\begin{tabular}{|c|c|}
\hline Title & Quantum reaction boundary to mediate reactions in laser fields \\
\hline Author(s) & Kawai, Shinnosuke; Komatsuzaki, Tamiki \\
\hline Citation & $\begin{array}{l}\text { Journal of Chemical Physics, 134(2), } 024317 \\
\text { https://doi.org/10.1063/1.3528937 }\end{array}$ \\
\hline Issue Date & 2011-01-14 \\
\hline Doc URL & http:/hdl.handle.net/2115/44992 \\
\hline Rights & $\begin{array}{l}\text { Copyright } 2011 \text { A merican Institute of Phy sics. This article may be downloaded for personal use only. A ny other use } \\
\text { requires prior permission of the author and the A merican Institute of Physics. The following article appeared in J. } \\
\text { Chem. Phys. 134, } 024317 \text { (2011) and may be found at https://dx.doi.org/10.1063/1.3528937 }\end{array}$ \\
\hline Type & article \\
\hline File Information & JCP1342_024317.pdf \\
\hline
\end{tabular}

Instructions for use 


\title{
Quantum reaction boundary to mediate reactions in laser fields
}

\author{
Shinnosuke Kawaia) and Tamiki Komatsuzaki \\ Molecule \& Life Nonlinear Sciences Laboratory, Research Institute for Electronic Science, \\ Hokkaido University, Kita 20 Nishi 10, Kita-ku, Sapporo 001-0020, Japan
}

(Received 25 October 2010; accepted 1 December 2010; published online 12 January 2011)

\begin{abstract}
Dynamics of passage over a saddle is investigated for a quantum system under the effect of timedependent external field (laser pulse). We utilize the recently developed theories of nonlinear dynamics in the saddle region, and extend them to incorporate both time-dependence of the external field and quantum mechanical effects of the system. Anharmonic couplings and laser fields with any functional form of time dependence are explicitly taken into account. As the theory is based on the Weyl expression of quantum mechanics, interpretation is facilitated by the classical phase space picture, while no "classical approximation" is involved. We introduce a quantum reactivity operator to extract the reactive part of the system. In a model system with an optimally controlled laser field for the reaction, it is found that the boundary of the reaction in the phase space, extracted by the reactivity operator, is modulated with time by the effect of the laser field, to "catch" the system excited in the reactant region, and then to "release" it into the product region. This method provides new insights in understanding the origin of optimal control of chemical reactions by laser fields. (C) 2011 American Institute of Physics. [doi:10.1063/1.3528937]
\end{abstract}

\section{INTRODUCTION}

Controlling chemical reactions to yield desired products by external fields has been one of the most intriguing subjects in these several decades. The recent development in optical laser technology ${ }^{1-7}$ has enabled us to control chemical reactions by laser fields that are strong enough to change the underlying potential energy surface of a molecule at the time scale of the molecular motion, i.e., pico- or femtosecond. By properly designing the pulse shape, one can guide the system to make the transition to the desired product channel. Theoretical algorithms to obtain the optimally designed pulse shape have been well developed. ${ }^{1,3,8-14}$ The optimal pulse is given as an automatic output from those algorithms and often has a complicated functional form. It is desired to construct a framework to provide firm physical interpretation about why that particular pulse shape leads to the target product channel.

In many reactions, the potential energy surface has a saddle point between the reactant and the product regions. The possibility for the system to have a chemical reaction is primarily determined by the ability to surmount the reaction barrier in the region around the saddle. Recently there has been a great progress in the study of dynamics around a rank-one saddle point ${ }^{15-39}$ in terms of the geometrical structure of the phase space. There two kinds of important objects play pivotal roles in understanding the occurrence of reactions. One is what chemists have termed "transition state (TS)" $" 40-47$ which was originally defined by Wigner ${ }^{41}$ as a surface dividing the phase space into two distinct regions, i.e., reactant and product, so that the system starting from the reactant must cross this surface once and only once before being captured in

a)Electronic mail: skawai@es.hokudai.ac.jp. Research Fellow of the Japan Society for the Promotion of Science. the product. In other words, the TS separates the space into "before" and "after" the reaction. The other important building block of the phase space in determining the fate of the reaction are so-called invariant manifolds. ${ }^{15,16}$ An invariant manifold is a set of phase space points such that, once the system is in that manifold, the system will stay in it perpetually. If the dimension of the manifold is less than that of the phase space by one, the manifold can divide the space into two disjoint regions. [Recall that a one-dimensional object (e.g., a line or a curve) can divide a two-dimensional space (e.g., a plane) into two, but not a three-dimensional space.] Due to the invariance of the manifold, no trajectory can cross it from one side to the other. One of the most important invariant manifolds in chemical reactions is that which separates the trajectories going to the product and those going to the reactant. Then, once we know which side of the manifold a given initial condition is, we can immediately know, without any trajectory calculations, whether the system goes to the product or not.

Recent studies ${ }^{15-24,26,32-39,48-52}$ have revealed that, even under the existence of anharmonic couplings among the modes, ${ }^{15-24,26,39,48-51}$ time-dependent external fields such as laser fields, ${ }^{32,33}$ and even stochastic random force exerted by solvents, ${ }^{27-31,34-38}$ there robustly exists a reaction coordinate, at least locally in the saddle region in the phase space, whose motion is decoupled from all the other (nonreactive) coordinates. The mathematical technique to construct this coordinate transformation is called normal form (NF) theory. In terms of this new coordinate, we can easily extract the no-return TS and the invariant manifolds to separate the destination of the reaction, i.e., either to the reactant or the product. These theories have been applied not only to chemical reactions, ${ }^{48-51}$ but also to, for example, ionization of a hydrogen atom in crossed electric and magnetic fields, ${ }^{21,22,39}$ isomerization of clusters, ${ }^{19,20,23}$ and the escape of asteroids 
from Mars. ${ }^{52}$ However, most studies on reaction dynamics from the viewpoint of the underlying phase space geometry have been devoted only to classical systems. The generalization of the theories to quantum systems is highly required for the control of chemical reactions to the desired product states.

Quantum mechanical version of the NF theory has been developed in the semiclassical level ${ }^{53-55}$ as well as in the Wigner-Weyl formalism. ${ }^{56-58}$ The quantum mechanical analogue of NF theory is called Van Vleck perturbation theory. ${ }^{17,18,59}$ In place of the coordinate transformation, one applies a unitary transformation by which the Hamiltonian is made as nearly diagonal as possible. In the Wigner-Weyl formalism in Refs. 56-58, the calculations are performed by using the phase space representations which provides a unique correspondence to quantum mechanical operators, and exactly reproduce the result of the quantum Van Vleck perturbation theory. The motivation for using the Wigner-Weyl formalism is that the lowest-order approximation in $\hbar$ corresponds to the classical NF, facilitating the study of quantumclassical correspondence, ${ }^{56}$ and that functions are much easier to handle than operators. ${ }^{57}$

In this paper, we present in Sec. II a quantum mechanical and time-dependent generalization of the reaction dynamics theories developed for classical systems. Particular attention is paid to the quantum counterpart of the (classical) concept of the invariant manifold (the separatrix) to uniquely separate the reactive and the nonreactive regions of the phase space. Then, we present a numerical illustration of how the separatrix moves by the effect of the optimal laser pulse in a simple model system in Sec. III. The main result is that we can find, up to a certain high energy, a quantum reactivity operator which extracts the reactive part of the system. The reaction boundary, the reactive and nonreactive regions in the phase space, moves with time, modulated by the laser field so that the boundary "catches" the system excited in the reactant and "releases" it into the product, resulting in increase of the reaction probability. Finally, we give the conclusion and outlook in Sec. IV.

\section{THEORY}

In this section we start our discussion with a simple classical system of a parabolic barrier with no coupling among the modes (Sec. II A). There we find a clear boundary that divides the whole phase space into a region to bring the system to the reactant and that to the product. (One can refer to books ${ }^{15,16}$ and papers ${ }^{17-24,26,39}$ for the generalization to nonlinearly coupled multidimensional classical systems.) For the sake of completeness, we give a brief review of the Wigner-Weyl formalism of quantum mechanics in Sec. II B. Then we consider the quantum system of the parabolic barrier by the Wigner-Weyl formalism, and discuss the quantum counterpart of the classical phase space reaction boundary in Sec. II C. The effects of anharmonicity and the external laser field are incorporated by use of quantum NF theory in Sec. II D.

\section{A. Classical dynamics of passage over a parabolic barrier}

When the total energy of the system is only slightly above the saddle point energy, the Hamiltonian can be approximated by

$$
H(\boldsymbol{q}, \boldsymbol{p})=\frac{p_{1}{ }^{2}}{2}-\frac{\lambda^{2}}{2} q_{1}^{2}+\sum_{\ell=2}^{n}\left(\frac{p_{\ell}{ }^{2}}{2}+\frac{\omega_{\ell}{ }^{2}}{2} q_{\ell}{ }^{2}\right),
$$

by introducing the so-called normal mode coordinates $\left(q_{1}, \ldots, q_{n}\right)$, which diagonalize the potential energy, and their conjugate momenta $\left(p_{1}, \ldots, p_{n}\right)$. We have assigned the unstable direction as mode 1 , with the curvature of the potential $-\lambda^{2} / 2$. The other modes are vibrational modes with frequencies $\omega_{\ell}$. The origin $\boldsymbol{q}=\mathbf{0}$ of the coordinates is taken at the position of the saddle point.

The following set of variables is often useful in the calculation:

$x_{1}=\frac{\lambda q_{1}+p_{1}}{\sqrt{2 \lambda}}, \quad \xi_{1}=\frac{p_{1}-\lambda q_{1}}{\sqrt{2 \lambda}}$,

$x_{\ell}=\frac{\omega_{\ell} q_{\ell}-\mathrm{i} p_{\ell}}{\sqrt{2 \omega_{\ell}}}, \quad \xi_{\ell}=\frac{p_{\ell}-\mathrm{i} \omega_{\ell} q_{\ell}}{\sqrt{2 \omega_{\ell}}} \quad(\ell=2, \ldots, n)$.

For the reactive mode, $\left(x_{1}, \xi_{1}\right)$ defines a skewed coordinate system in the phase space as in Fig. 1, whereas for the nonreactive modes $\left(x_{\ell}, \xi_{\ell}\right)$ take complex values. The product of $x_{\ell}$ and $\xi_{\ell}$ is called action variable:

$$
\begin{aligned}
I_{1} & \stackrel{\text { def }}{=} x_{1} \xi_{1}=\frac{p_{1}^{2}}{2 \lambda}-\frac{\lambda}{2} q_{1}{ }^{2}, \\
I_{\ell} \stackrel{\text { def }}{=} \mathrm{i} x_{\ell} \xi_{\ell} & =\frac{p_{\ell}{ }^{2}}{2 \omega_{\ell}}+\frac{\omega_{\ell}}{2} q_{\ell}^{2} \quad(\ell=2, \ldots, n) .
\end{aligned}
$$

Then the Hamiltonian of Eq. (1) is rewritten in terms of the action variables:

$$
H=\lambda I_{1}+\sum_{\ell=2}^{n} \omega_{\ell} I_{\ell}
$$

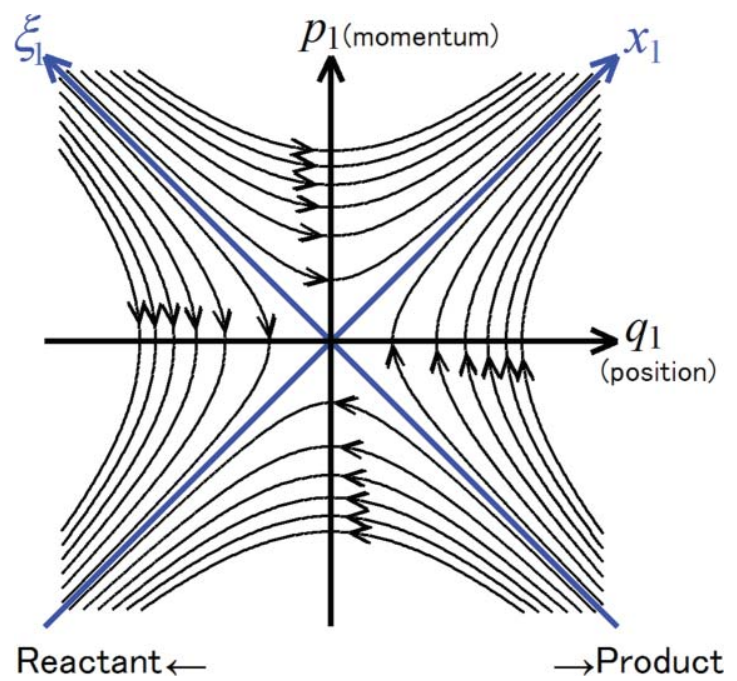

FIG. 1. Phase space flow in the saddle region, for the case of harmonic approximation. 
It is easily seen that the actions are constants of motion $\left(\mathrm{d} I_{1} / \mathrm{d} t=0, \mathrm{~d} I_{\ell} / \mathrm{d} t=0\right)$ under the Hamiltonian given by Eq. (1). Therefore the trajectories run along the hyperbolas given by $I_{1}=$ const. as shown in Fig. 1 .

Suppose that $q_{1}=-\infty$ corresponds to the reactant (i.e., before the reaction), and $q_{1}=+\infty$ to the product (after the reaction). The trajectories with $x_{1}>0$ and $\xi_{1}>0$ are "forward reactive" trajectories because they climb up from the reactant region, overcome the barrier, and go down to the product region (i.e., the reaction occurs). On the other hand, the trajectories with $x_{1}<0$ and $\xi_{1}>0$ are "forward nonreactive" trajectories because they start in the reactant region, but are reflected by the barrier, and goes back into the reactant region. The trajectories with $x_{1}<0$ and $\xi_{1}<0$ are "backward reactive" trajectories, because they climb up from the product region and go down the barrier to the reactant region (i.e., the backward reaction). Similarly, the trajectories with $x_{1}>0$ and $\xi_{1}<0$ are "backward nonreactive" trajectories. Note that the sign of $x_{1}$ determines the fate of the system, that is, if $x_{1}$ is positive (negative), the trajectory will go to the product (reactant) side, without mattering where it initially was. The set $\left\{x_{1}=0\right\}$, thus divides all the climbing trajectories into two disjoint sets: ones going to the product and the others going to the reactant. Once we know the sign of $x_{1}$, we can tell the fate of the reaction without further observing the trajectory. In addition, once the system is located on the dividing surface of $x_{1}=0$, it stays there perpetually (i.e., the set $\left\{x_{1}=0\right\}$ forms an invariant manifold in the phase space).

\section{B. Wigner-Weyl representations of quantum mechanics}

To investigate the quantum counterpart of the phase space structure found in Sec. II A, we utilize the concepts of Wigner distribution and Weyl representation. ${ }^{60,61}$ The Weyl representation of a quantum mechanical operator $\hat{A}$ is a function in the classical phase space variables $(\boldsymbol{q}, \boldsymbol{p})$ given by

$A(\boldsymbol{q}, \boldsymbol{p})=\mathrm{Cl}[\hat{A}] \stackrel{\text { def }}{=} \int\left\langle\boldsymbol{q}-\frac{\boldsymbol{s}}{2}|\hat{A}| \boldsymbol{q}+\frac{\boldsymbol{s}}{2}\right\rangle \exp \left(\frac{\text { is } \cdot \boldsymbol{p}}{\hbar}\right) \mathrm{d} \boldsymbol{s}$,

where $|\boldsymbol{q} \pm \boldsymbol{s} / 2\rangle$ is the eigenstate of the position operator $\hat{\boldsymbol{q}}$ with eigenvalues $q \pm s / 2$. We have introduced the symbol $\mathrm{Cl}[\cdot]$ to denote the transformation from the quantum mechanical operator to the phase space function.

The backward transformation of the Weyl representation is called Weyl quantization, where the matrix element of the operator $\hat{A}$ corresponding to the classical quantity $A(\boldsymbol{q}, \boldsymbol{p})$ is given by

$$
\begin{aligned}
\left\langle\boldsymbol{q}^{\prime}|\hat{A}| \boldsymbol{q}^{\prime \prime}\right\rangle= & \left\langle\boldsymbol{q}^{\prime}|\mathrm{Op}[A]| \boldsymbol{q}^{\prime \prime}\right\rangle \\
\stackrel{\text { def }}{=} & (2 \pi \hbar)^{-n} \int A\left(\frac{\boldsymbol{q}^{\prime}+\boldsymbol{q}^{\prime \prime}}{2}, \boldsymbol{p}\right) \\
& \times \exp \left(\frac{\mathrm{i}\left(\boldsymbol{q}^{\prime}-\boldsymbol{q}^{\prime \prime}\right) \cdot \boldsymbol{p}}{\hbar}\right) \mathrm{d} \boldsymbol{p},
\end{aligned}
$$

where we have introduced the symbol Op[·] to denote the Weyl quantization of a classical function. Some elementary examples for the Weyl quantization can be obtained by tedious but straightforward calculations from Eq. (6):

$$
\begin{array}{lc}
\mathrm{Op}[q]=\hat{q}, & \mathrm{Op}\left[q^{2}\right]=\hat{q}^{2}, \\
\mathrm{Op}[p]=\hat{p}, & \mathrm{Op}\left[p^{2}\right]=\hat{p}^{2}, \\
\mathrm{Op}[q p]=(\hat{q} \hat{p}+\hat{p} \hat{q}) / 2=\hat{q} \hat{p}-\mathrm{i} \hbar / 2 .
\end{array}
$$

The Weyl representation of a density operator $|\psi\rangle\langle\psi|$, where $|\psi\rangle$ is a certain state, divided by $(2 \pi \hbar)^{n}$ is called Wigner distribution

$$
\begin{aligned}
\rho_{\psi}(\boldsymbol{q}, \boldsymbol{p})= & (2 \pi \hbar)^{-n} \mathrm{Cl}[|\psi\rangle\langle\psi|] \\
= & (2 \pi \hbar)^{-n} \int\left\langle\boldsymbol{q}-\frac{\boldsymbol{s}}{2} \mid \psi\right\rangle\left\langle\psi \mid \boldsymbol{q}+\frac{\boldsymbol{s}}{2}\right\rangle \\
& \times \exp \left(\frac{\mathrm{i} \cdot \boldsymbol{p}}{\hbar}\right) \mathrm{d} \boldsymbol{s} .
\end{aligned}
$$

A remarkable fact about the Weyl representation and the Wigner distribution is that the quantum mechanical expectation value is exactly given by the phase space integral:

$$
\langle\psi|\hat{A}| \psi\rangle=\int A(\boldsymbol{q}, \boldsymbol{p}) \rho_{\psi}(\boldsymbol{q}, \boldsymbol{p}) \mathrm{d} \boldsymbol{q} \mathrm{d} \boldsymbol{p} .
$$

Classical representation of the product of two operators $\hat{A}$ and $\hat{B}$ is called star-product: ${ }^{57,60}$

$$
\mathrm{Op}[A \star B]=\mathrm{Op}[A] \mathrm{Op}[B],
$$

whose explicit form is given by

$$
\begin{gathered}
A \star B \stackrel{\text { def }}{=} A \exp \left(\frac{\mathrm{i} \hbar \Lambda}{2}\right) B, \\
\Lambda \stackrel{\text { def }}{=} \overleftarrow{\partial_{q}} \cdot \overrightarrow{\partial_{p}}-\overleftarrow{\partial_{p}} \cdot \overrightarrow{\partial_{q}},
\end{gathered}
$$

where the arrows indicate whether the partial differentiation acts on $A$ or $B$. The first two terms in the expansion in $\hbar$ are

$$
A \star B=A B+\frac{\mathrm{i} \hbar}{2}\{A, B\}+O\left(\hbar^{2}\right),
$$

where $\{\cdot, \cdot\}$ denotes the (classical) Poisson bracket. That is, the star product is given by a simple product $A B$ plus correction terms of order $\hbar$ and higher orders.

The commutator of operators (divided by a constant i $\hbar$ ) is represented by the so-called Moyal bracket

$$
\{\{A, B\}\} \stackrel{\text { def }}{=} \frac{1}{\mathrm{i} \hbar}(A \star B-B \star A),
$$

that is,

$$
\mathrm{Op}\left[\{\{A, B\}]=\frac{1}{\mathrm{i} \hbar}(\hat{A} \hat{B}-\hat{B} \hat{A})=\frac{1}{\mathrm{i} \hbar}[\hat{A}, \hat{B}] .\right.
$$

By using Eq. (11), we have

$$
\begin{aligned}
\{\{A, B\}\} & =\frac{2}{\hbar} A \sin \left(\frac{\hbar \Lambda}{2}\right) B, \\
& =\{A, B\}+O\left(\hbar^{2}\right) .
\end{aligned}
$$

Thus Moyal bracket simply corresponds to Poisson bracket at the classical limit $\hbar \rightarrow 0$ and the correction terms start from the second-order $\hbar^{2}$. To express the exponential of an operator

$$
\exp (\hat{A})=\sum_{n=0}^{\infty} \frac{1}{n !} \hat{A}^{n}
$$


in the Weyl classical expression, the star-exponential is defined

$$
\exp _{\star}(A) \stackrel{\text { def }}{=} \sum_{n=0}^{\infty} \frac{1}{n !} \underbrace{A \star A \star \cdots \star A}_{n},
$$

that is,

$$
\mathrm{Op}\left[\exp _{\star}(A)\right]=\exp (\mathrm{Op}[A]) .
$$

\section{Quantum dynamics of passage over a parabolic barrier}

We now consider the quantum mechanics for the parabolic barrier. The Hamiltonian operator is quantized from Eq. (1)

$$
\begin{aligned}
\hat{H} & =\mathrm{Op}[H] \\
& =\frac{\hat{p}_{1}^{2}}{2}-\frac{\lambda^{2}}{2} \hat{q}_{1}^{2}+\sum_{\ell=2}^{n}\left(\frac{\hat{p}_{\ell}^{2}}{2}+\frac{\omega_{\ell}^{2}}{2} \hat{q}_{\ell}^{2}\right) .
\end{aligned}
$$

Quantum mechanical action operator is defined by quantizing Eq. (3):

$$
\hat{I}_{1}=\frac{\hat{p}_{1}^{2}}{2 \lambda}-\frac{\lambda}{2} \hat{q}_{1}^{2}
$$

It is now seen that the action operator $\hat{I}_{1}$ commutes with the Hamiltonian: $\left[\hat{I}_{1}, \hat{H}\right]=0$. This means that the eigenstates of $\hat{H}$ can be taken to be eigenstates of $\hat{I}_{1}$ simultaneously.

The eigenstate wavefunctions of $\hat{I}_{1}$ are obtained analytically. ${ }^{57,62}$ There are two linearly independent solutions for the same eigenvalue. One can take the following basis set for this two-dimensional eigenspace of $\hat{I}_{1}$ :

$$
\begin{aligned}
\Phi_{\alpha, \mathrm{i}: \mathrm{r}}\left(q_{1}\right)= & \frac{\exp (\pi \alpha / 4)}{\sqrt{2} \pi(2 \hbar)^{1 / 4}} \Gamma\left(\frac{1}{2}-\mathrm{i} \alpha\right) \\
& \times D_{-1 / 2+\mathrm{i} \alpha}\left(\exp (-\mathrm{i} \pi / 4) \sqrt{2 / \hbar} q_{1}\right), \\
\Phi_{\alpha, \mathrm{i}: \mathrm{p}}\left(q_{1}\right)= & \frac{\exp (\pi \alpha / 4)}{\sqrt{2} \pi(2 \hbar)^{1 / 4}} \Gamma\left(\frac{1}{2}-\mathrm{i} \alpha\right) \\
& \times D_{-1 / 2+\mathrm{i} \alpha}\left(-\exp (-\mathrm{i} \pi / 4) \sqrt{2 / \hbar} q_{1}\right),
\end{aligned}
$$

where the suffix $\alpha$ denotes the eigenvalue divided by $\hbar$ :

$$
\begin{aligned}
& \hat{I}_{1}\left|\Phi_{\alpha, \mathrm{i}: \mathrm{r}}\right\rangle=\alpha \hbar\left|\Phi_{\alpha, \mathrm{i}: \mathrm{r}}\right\rangle, \\
& \hat{I}_{1}\left|\Phi_{\alpha, \mathrm{i}: \mathrm{p}}\right\rangle=\alpha \hbar\left|\Phi_{\alpha, \mathrm{i}: \mathrm{p}}\right\rangle .
\end{aligned}
$$

The symbol $\Gamma$ denotes the gamma function and $D$ the parabolic cylinder function. ${ }^{63}$ The normalization is taken to satisfy the orthonormality and the completeness relations:

$$
\begin{aligned}
& \left\langle\Phi_{\alpha, \mathrm{i}: \mathrm{r}} \mid \Phi_{\beta, \mathrm{i}: \mathrm{r}}\right\rangle=\delta(\alpha-\beta), \\
& \left\langle\Phi_{\alpha, \mathrm{i}: \mathrm{p}} \mid \Phi_{\beta, \mathrm{i}: \mathrm{p}}\right\rangle=\delta(\alpha-\beta), \\
& \left\langle\Phi_{\alpha, \mathrm{i}: \mathrm{p}} \mid \Phi_{\beta, \mathrm{i}: \mathrm{r}}\right\rangle=0, \\
& \int \mathrm{d} \alpha\left(\left|\Phi_{\alpha, \mathrm{i}: \mathrm{r}}\right\rangle\left\langle\Phi_{\alpha, \mathrm{i}: \mathrm{r}}|+| \Phi_{\alpha, \mathrm{i}: \mathrm{p}}\right\rangle\left\langle\Phi_{\alpha, \mathrm{i}: \mathrm{p}}\right|\right)=1 .
\end{aligned}
$$

From the asymptotic behavior of the parabolic cylinder functions ${ }^{63}$ it is seen that $\Phi_{\alpha, \text { i:r }}$ contains the incoming wave only in the reactant region $\left(q_{1} \rightarrow-\infty\right)$, while it has both transmitted wave in the product side and the reflected wave in the reactant side. Similarly, $\Phi_{\alpha, \text { i:p }}$ has the incoming wave only in the product region, the outgoing wave in both regions. Thus the suffix "i:r" stands for "incoming from reactant" and "i:p" for "incoming from product," following the notation of Ref. 57.

By taking linear combinations of $\Phi_{\alpha, \text { i:r }}$ and $\Phi_{\alpha, \text { i:p }}$, we can form another basis set as follows:

$$
\begin{aligned}
& \Phi_{\alpha, \mathrm{o}: \mathrm{r}}\left(q_{1}\right)=\frac{\exp (\mathrm{i} \pi / 4)}{\sqrt{2 \pi}} \Gamma\left(\frac{1}{2}+\mathrm{i} \alpha\right)\left(\exp (-\pi \alpha / 2) \Phi_{\alpha, \mathrm{i}: \mathrm{r}}\left(q_{1}\right)\right. \\
& \left.-\mathrm{i} \exp (\pi \alpha / 2) \Phi_{\alpha, \mathrm{i}: \mathrm{p}}\left(q_{1}\right)\right) \\
& =\frac{\exp (\pi \alpha / 4)}{\sqrt{2} \pi(2 \hbar)^{1 / 4}} \Gamma\left(\frac{1}{2}+\mathrm{i} \alpha\right) \\
& \times D_{-1 / 2-\mathrm{i} \alpha}\left(\exp (\mathrm{i} \pi / 4) \sqrt{2 / \hbar} q_{1}\right), \\
& \Phi_{\alpha, \mathrm{o}: \mathrm{p}}\left(q_{1}\right)=\frac{\exp (\mathrm{i} \pi / 4)}{\sqrt{2 \pi}} \Gamma\left(\frac{1}{2}+\mathrm{i} \alpha\right)\left(-\mathrm{i} \exp (\pi \alpha / 2) \Phi_{\alpha, \mathrm{i}: \mathrm{r}}\left(q_{1}\right)\right. \\
& \left.+\exp (-\pi \alpha / 2) \Phi_{\alpha, \mathrm{i}: \mathrm{p}}\left(q_{1}\right)\right) \\
& =\frac{\exp (\pi \alpha / 4)}{\sqrt{2} \pi(2 \hbar)^{1 / 4}} \Gamma\left(\frac{1}{2}+\mathrm{i} \alpha\right) \\
& \times D_{-1 / 2-\mathrm{i} \alpha}\left(-\exp (\mathrm{i} \pi / 4) \sqrt{2 / \hbar} q_{1}\right),
\end{aligned}
$$

The suffix "o:r" and "o:p" here stand for "outgoing to reactant" and "outgoing to product," respectively. The function $\Phi_{\alpha, \text { orr }}$ has outgoing wave only in the reactant side, while it has incoming waves both in the reactant and the product sides. Similarly, the function $\Phi_{\alpha, \text { o:p }}$ has outgoing wave only in the product side.

Suppose we are given a state $|\psi(t)\rangle$ at time $t$, and ask what is the probability that the system goes to the product side for $t \rightarrow+\infty$. The answer is given by taking a projection of $|\psi(t)\rangle$ onto the outgoing-to-product state $\left|\Phi_{\alpha, \text { o:p }}\right\rangle$, and integrating over all the possible values of $\alpha$. Thus the reaction probability is

$$
P_{\text {reaction }}=\int \mathrm{d} \alpha\left|\left\langle\Phi_{\alpha, \mathrm{o}: \mathrm{p}} \mid \psi(t)\right\rangle\right|^{2} \text {. }
$$

As has been introduced by Miller, ${ }^{64}$ we here define a projection operator

$$
\hat{\mathscr{P}}_{0} \stackrel{\text { def }}{=} \int \mathrm{d} \alpha\left|\Phi_{\alpha, \mathrm{o}: \mathrm{p}}\right\rangle\left\langle\Phi_{\alpha, \mathrm{o}: \mathrm{p}}\right|,
$$

with which the reaction probability is given as the expectation value

$$
\begin{aligned}
P_{\text {reaction }} & =\left\langle\psi(t)\left|\hat{\mathscr{P}}_{0}\right| \psi(t)\right\rangle \\
& =\int \rho_{\psi}(\boldsymbol{q}, \boldsymbol{p}, t) \mathscr{P}_{0}(\boldsymbol{q}, \boldsymbol{p}) \mathrm{d} \boldsymbol{q} \mathrm{d} \boldsymbol{p},
\end{aligned}
$$

where $\rho_{\psi}$ is the Wigner distribution for $\psi(t)$, and $\mathscr{P}_{0}$ is the Weyl classical representation of $\hat{\mathscr{P}}_{0}$ [see Eq. (9)]. 
From Eq. (26), we have

$$
\begin{aligned}
\mathscr{P}_{0}(\boldsymbol{q}, \boldsymbol{p}) & =\mathrm{Cl}\left[\hat{\mathscr{P}}_{0}\right] \\
& =\int \mathrm{d} \alpha \mathrm{Cl}\left[\left|\Phi_{\alpha, 0: \mathrm{p}}\right\rangle\left\langle\Phi_{\alpha, \mathrm{o}: \mathrm{p}}\right|\right] \\
& =\int \mathrm{d} \alpha(2 \pi \hbar)^{n} \rho_{\alpha, \mathrm{o}: \mathrm{p}}(\boldsymbol{q}, \boldsymbol{p}),
\end{aligned}
$$

with the Wigner distribution $\rho_{\alpha, \mathrm{o}: \mathrm{p}}(\boldsymbol{q}, \boldsymbol{p})$ of the scattering eigenstate $\Phi_{\alpha, \text { o:p }}$ [see Eq. (8)]. The Wigner distributions of the scattering eigenstates were calculated in Ref. 62. Integration over $\alpha$ leads to a surprisingly simple result:

$$
\mathscr{P}_{0}(\boldsymbol{q}, \boldsymbol{p})=\Theta\left(x_{1}\right),
$$

where $x_{1}$ is defined in Eq. (2) (see also Fig. 1), and $\Theta$ is the Heaviside step function

$$
\Theta\left(x_{1}\right)= \begin{cases}1 & \left(x_{1}>0\right) \\ 0 & \left(x_{1}<0\right) .\end{cases}
$$

Recall that the phase space region with $x_{1}>0$ is the trajectories going to the product side in the classical case. The result of Eq. (29) combined with Eq. (27) tells us that we can draw the same picture in quantum mechanical case: given the Wigner distribution in the phase space, we take only the part $x_{1}>0$ and integrate the distribution. The result gives the probability that the system goes to the product side. A similar result was presented by Balazs and Voros. ${ }^{62}$ The reactive part is given by $x_{1}>0$ even when there is quantum mechanical tunneling. They showed that the Wigner representation interprets the tunneling phenomenon as due to broadening of the distribution into the positive energy region, even when the energy eigenvalue is negative. In summary, the picture that the region $x_{1}>0$ is the reactive part holds exactly in the quantum harmonic system. (Here we use the word "reactive" to mean "going to the product," though the strict meaning of the reaction may be "going from the reactant to the product.") The phase space boundary of the reactivity is therefore given by $x_{1}=0$, just the same as the classical mechanics.

\section{Time-dependent quantum normal form theory for incorporating nonlinearities and external field}

The findings for the parabolic barrier case in Sec. II C can be used as the starting point for more general cases. Here we treat the effects of anharmonicities and time-dependent external field perturbatively by normal form (NF) theory. Suppose the Schrödinger equation

$$
\mathrm{i} \hbar \frac{\partial}{\partial t}|\psi(t)\rangle=\hat{H}(t)|\psi(t)\rangle .
$$

The basic idea of the NF theory is the transformation of the Hamiltonian to make it commute with the action operator $\hat{I}_{1}$ by introducing a unitary transformation $\hat{U}(t)$ to the wavefunction

$$
\left|\psi^{\prime}(t)\right\rangle=\hat{U}(t)|\psi(t)\rangle,
$$

which can be given by using a Hermitian operator $\hat{F}(t)$

$$
\hat{U}(t)=\exp \left(\frac{\mathrm{i}}{\hbar} \hat{F}(t)\right) .
$$

The transformed wavefunction obeys the Schrödinger equation

$$
\mathrm{i} \hbar \frac{\partial}{\partial t}\left|\psi^{\prime}(t)\right\rangle=\hat{H}^{\prime}(t)\left|\psi^{\prime}(t)\right\rangle
$$

with the transformed Hamiltonian

$$
\hat{H}^{\prime}(t)=\hat{U}(t) \hat{H}(t) \hat{U}(t)^{\dagger}+\mathrm{i} \hbar \frac{\partial \hat{U}(t)}{\partial t} \hat{U}(t)^{\dagger},
$$

where $\dagger$ denotes Hermitian conjugate. With the technique which will be presented in this section, we can determine the form of $\hat{U}(t)$ [or equivalently $\hat{F}(t)$ ] to make the new Hamiltonian commute with $\hat{I}_{1}$ :

$$
\left[\hat{H}^{\prime}(t), \hat{I}_{1}\right]=0 .
$$

This allows us to follow the procedure discussed for a parabolic barrier system in Sec. II C. Namely, the eigenstates of $\hat{I}_{1}$, whose wavefunctions (and hence $\hat{\mathscr{P}}_{0}$ ) can be obtained analytically, are also those of the new Hamiltonian $\hat{H}^{\prime}(t)$. The reaction probability is thus given by

$$
P_{\text {reaction }}=\left\langle\psi^{\prime}(t)\left|\hat{\mathscr{P}}_{0}\right| \psi^{\prime}(t)\right\rangle \text {. }
$$

In terms of the original wavefunction this becomes

$P_{\text {reaction }}=\left\langle\psi(t)\left|\hat{U}(t)^{\dagger} \hat{\mathscr{P}}_{0} \hat{U}(t)\right| \psi(t)\right\rangle=\langle\psi(t)|\hat{\mathscr{P}}| \psi(t)\rangle$,

where

$$
\hat{\mathscr{P}} \stackrel{\text { def }}{=} \hat{U}(t)^{\dagger} \hat{\mathscr{P}}_{0} \hat{U}(t) .
$$

We call $\hat{\mathscr{P}}$ anharmonic reactivity operator because it takes into account anharmonicities (and time-dependent external field).

\section{Expansion of Hamiltonian}

In order to calculate the unitary transformation, we first expand the Hamiltonian in the form of power series. Hereafter it is convenient to write all the operators in the Weyl classical representation. Following Ref. 32, let (the Weyl representation of) the Hamiltonian be

$$
H^{\text {tot }}(\boldsymbol{q}, \boldsymbol{p}, t)=H^{\text {sys }}(\boldsymbol{q}, \boldsymbol{p})+H^{\mathrm{ex}}(\boldsymbol{q}, \boldsymbol{p}, t),
$$

where $H^{\text {sys }}$ is the Hamiltonian of an isolated molecule and $H^{\mathrm{ex}}$ describes the interaction with the external laser field. We introduce a formal perturbation parameter $\varepsilon$ which will be set equal to one after all the calculations:

$H^{\mathrm{sys}}(\boldsymbol{q}, \boldsymbol{p})=\sum_{v=0}^{\infty} \varepsilon^{v} H_{v}^{\mathrm{sys}}(\boldsymbol{q}, \boldsymbol{p})$,

$H^{\mathrm{ex}}(\boldsymbol{q}, \boldsymbol{p}, t)=\sum_{\nu=0}^{\infty} \varepsilon^{\nu} H_{v}^{\mathrm{ex}}(\boldsymbol{q}, \boldsymbol{p}, t)$,

$H_{v}^{\text {sys }}(\boldsymbol{q}, \boldsymbol{p})=\sum_{\sum_{\ell}\left(j_{\ell}+k_{\ell}\right)=2+v} \alpha_{j k} q_{1}{ }^{j_{1}} \cdots q_{n}{ }^{j_{n}}{p_{1}}^{k_{1}} \cdots p_{n}{ }^{k_{n}}$,

$H_{v}^{\mathrm{ex}}(\boldsymbol{q}, \boldsymbol{p}, t)=\sum_{\sum_{\ell}\left(j_{\ell}+k_{\ell}\right)=1+v} \beta_{j k}(t) q_{1}^{j_{1}} \cdots q_{n}^{j_{n}}{p_{1}}^{k_{1}} \cdots p_{n}{ }^{k_{n}}$. 
Thus the $v$ th-order term in $H^{\text {sys }}$ is a homogeneous polynomial of degree $2+v$, with the coefficients $\alpha_{j k}$. The $v$ th-order term in $H^{\mathrm{ex}}$ is a homogeneous polynomial of degree $1+v$, with the time-dependent coefficients $\beta_{j k}(t)$. The zeroth order of $H^{\text {sys }}$ coincides with the harmonic approximation [Eq. (1)]. Note that this order assignment of $\varepsilon^{v}$ may not be unique, but it was found that it results in appropriate normal form transformations in similar problems. ${ }^{34-37}$

When the kinetic energy does not depend on $\boldsymbol{q}, H^{\text {sys }}$ is just the same with the classical Hamiltonian. When the metric tensor is a function of $\boldsymbol{q}$, the Weyl representation $H^{\text {sys }}$ is different from the classical limit due to noncommutativity of $\boldsymbol{p}$ and $\boldsymbol{q}$ [e.g., Eq. (7)]. In the latter case, we can expand the kinetic energy in a polynomial of $\boldsymbol{q}$, and put the lowest-order terms (without $\boldsymbol{q}$ ) into $H_{0}^{\text {sys }}$ and higher-order terms into $H_{1}^{\text {sys }}$, $H_{2}^{\text {sys }}, \ldots$

Now we combine $H^{\text {sys }}$ and $H^{\text {ex }}$ to form the total Hamiltonian

$$
H^{\mathrm{tot}}=\sum_{\nu=0}^{\infty} \varepsilon^{\nu} H_{\nu},
$$

where

$$
\begin{aligned}
H_{v} & =H_{v}^{\mathrm{sys}}+H_{v}^{\mathrm{ex}} \\
H_{0} & =H_{0}^{\mathrm{sys}}+H_{0}^{\mathrm{ex}} \\
& =\lambda x_{1} \xi_{1}+\sum_{\ell=2}^{n} \mathrm{i} \omega_{\ell} x_{\ell} \xi_{\ell}+\sum_{\ell=1}^{n} b_{\ell}(t) x_{\ell}+\sum_{\ell=1}^{n} \beta_{\ell}(t) \xi_{\ell} .
\end{aligned}
$$

Here we have used the coordinate $(\boldsymbol{x}, \boldsymbol{\xi})$ defined in Eq. (2), and the time-dependent coefficients $b_{\ell}(t)$ and $\beta_{\ell}(t)$ come from $H_{0}^{\mathrm{ex}}$.

The unitary transformation is constructed by two steps, i.e., time-dependent shift and nonlinear transformations, which will be presented below.

\section{Transformation 1: Time-dependent shift}

The first step is to remove the time-dependent linear terms in $H_{0}$, which can be achieved by

$$
\begin{aligned}
U^{(0)}(t) & =\exp _{\star}\left(\frac{\mathrm{i}}{\hbar} F_{0}(t)\right) \\
F_{0}(t)= & \sum_{\ell=1}^{n}\left(-\xi_{\ell}^{\ddagger}(t) x_{\ell}+x_{\ell}^{\ddagger}(t) \xi_{\ell}\right. \\
& \left.+\frac{1}{2} \int^{t}\left(b_{\ell}(t) x_{\ell}^{\ddagger}(t)+\beta_{\ell}(t) \xi_{\ell}^{\ddagger}(t)\right) \mathrm{d} t\right)
\end{aligned}
$$

[for the first equation, see Eqs. (17) and (33)]. Here, the timedependent coefficients $x_{\ell}^{\ddagger}(t)$ and $\xi_{\ell}^{\ddagger}(t)$ ( $\ddagger$ must not be confused with Hermitian conjugate $\dagger$ ) are so-called transition state trajectory ${ }^{32,33}$ defined by

$$
\begin{array}{rlrl}
x_{1}^{\ddagger}(t)= & S\left[\lambda, \beta_{1}\right](t), & & \xi_{1}^{\ddagger}(t)=-S\left[-\lambda, b_{1}\right](t), \\
x_{\ell}^{\ddagger}(t)= & S\left[\mathrm{i} \omega_{\ell}, \beta_{\ell}\right](t), & & \xi_{\ell}^{\ddagger}(t)=-S\left[-\mathrm{i} \omega_{\ell}, b_{\ell}\right](t), \\
& (\ell=2, \ldots, n), &
\end{array}
$$

where

$$
S[\mu, f](t) \stackrel{\text { def }}{=} \frac{1}{(2 \pi)^{1 / 2}} \int_{-\infty}^{+\infty} \frac{\hat{f}(\omega)}{-\mu+\mathrm{i} \omega} \exp (\mathrm{i} \omega t) \mathrm{d} \omega
$$

for a complex number $\mu$ and a function $f(t)$, with the Fourier transform

$$
f(t)=\frac{1}{(2 \pi)^{1 / 2}} \int_{-\infty}^{+\infty} \hat{f}(\omega) \exp (\mathrm{i} \omega t) \mathrm{d} \omega .
$$

When $\mu$ is purely imaginary, the integral of Eq. (46) is taken as the principal value. ${ }^{32}$ The functional $S$ satisfies the following differential equation:

$$
\left(\frac{\mathrm{d}}{\mathrm{d} t}-\mu\right) S[\mu, f](t)=f(t) .
$$

From Eq. (35), the transformed Hamiltonian is given by

$$
H^{(0)} \stackrel{\text { def }}{=} U^{(0)} \star H^{\text {tot }} \star U^{(0)^{*}}+\mathrm{i} \hbar \frac{\partial U^{(0)}}{\partial t} \star U^{(0)^{*}},
$$

because the Hermitian conjugate of an operator becomes the complex conjugate in the Weyl representation. When the generating function $\left(F_{0}\right)$ is linear in the variables $(\boldsymbol{x}$ and $\boldsymbol{\xi})$ as in Eq. (44), the first term of the transformation takes a simple form: ${ }^{57,60}$

$$
U^{(0)} \star H^{\mathrm{tot}}(\boldsymbol{x}, \boldsymbol{\xi}, t) \star U^{(0) *}=H^{\mathrm{tot}}\left(\boldsymbol{x}+\boldsymbol{x}^{\ddagger}(t), \boldsymbol{\xi}+\boldsymbol{\xi}^{\ddagger}(t), t\right) .
$$

In other words, the transformation $U^{(0)}$ induces a shift of the coordinates by $\left(\boldsymbol{x}^{\ddagger}(t), \boldsymbol{\xi}^{\ddagger}(t)\right)$.

Inserting $H^{\text {tot }}=\sum_{\nu} \varepsilon^{v} H_{\nu}$ [Eq. (42)] into Eq. (49), and dividing the terms as

$$
\begin{aligned}
& H_{0}^{(0)}=U^{(0)} \star H_{0} \star U^{(0)^{*}}+\mathrm{i} \hbar \frac{\partial U^{(0)}}{\partial t} \star U^{(0)^{*}}, \\
& H_{v}^{(0)}=U^{(0)} \star H_{\nu} \star U^{(0)^{*}} \quad(v \geq 1),
\end{aligned}
$$

we have

$$
H^{(0)}=\sum_{\nu=0}^{\infty} \varepsilon^{v} H_{v}^{(0)}
$$

From Eqs. (44) and (50) we obtain

$$
\begin{aligned}
& H_{0}^{(0)}=\lambda x_{1} \xi_{1}+\sum_{\ell=2}^{n} \mathrm{i} \omega_{\ell} x_{\ell} \xi_{\ell}, \\
& H_{v}^{(0)}=H_{\nu}\left(\boldsymbol{x}+\boldsymbol{x}^{\ddagger}(t), \boldsymbol{\xi}+\boldsymbol{\xi}^{\ddagger}(t), t\right) \quad(v \geq 1) .
\end{aligned}
$$

Thus the time-dependent terms $b_{\ell}(t) x_{\ell}$ and $\beta_{\ell}(t) \xi_{\ell}$ in the zeroth-order part [Eq. (43)] have disappeared in Eq. (53) as desired.

\section{Transformation 2: Nonlinear transformations}

Next we treat the higher-order terms $H_{v}^{(0)}$ by successive operations of unitary transformations

$$
U=U^{(N)} \star \cdots \star U^{(2)} \star U^{(1)} \star U^{(0)},
$$


with

$$
U^{(\mu)}=\exp _{\star}\left(\frac{\mathrm{i}}{\hbar} \varepsilon^{\mu} F_{\mu}(t)\right) \quad(\mu \geq 0)
$$

( $U^{(0)}$ was described in Sec. II D 2). $U^{(\mu)}(\mu \geq 1)$ will be constructed so that the resultant transformed Hamiltonian commute with $\hat{I}_{1}$ up to the order $\varepsilon^{\mu}$ as follows.

Let

$$
H^{(\mu)} \stackrel{\text { def }}{=} U^{(\mu)} \star H^{(\mu-1)} \star U^{(\mu)^{*}}+\mathrm{i} \hbar \frac{\partial U^{(\mu)}}{\partial t} \star U^{(\mu)^{*}}
$$

be the transformed Hamiltonian at the $\mu$ th unitary transformation. For the series expansion of $H^{(\mu)}$

$$
H^{(\mu)}=\sum_{\nu=0}^{\infty} \varepsilon^{v} H_{v}^{(\mu)}
$$

by substituting Eqs. (56) and (57) to the left hand side of this equation, and equating them at each order of $\varepsilon$, we can obtain the following recursion formulas similarly to classical systems: ${ }^{32,57}$

$$
\begin{aligned}
H_{v}^{(\mu)}= & H_{v}^{(\mu-1)}+\sum_{s=1} \frac{1}{s !}\left(\operatorname{Mad}_{F_{\mu}}\right)^{s} H_{v-s \mu}^{(\mu-1)} \\
& -\sum_{s=1} \frac{1}{s !}\left(\operatorname{Mad}_{F_{\mu}}\right)^{s-1} \frac{\partial F_{\mu}}{\partial t} \delta_{v, s \mu}, \quad(v>\mu), \\
H_{\mu}^{(\mu)}= & H_{\mu}^{(\mu-1)}+\left\{\left\{H_{0}^{(0)}, F_{\mu}\right\}\right\}-\frac{\partial F_{\mu}}{\partial t}, \quad(v=\mu), \\
H_{v}^{(\mu)}= & H_{v}^{(\mu-1)}, \quad(v<\mu),
\end{aligned}
$$

where $\operatorname{Mad}_{F_{\mu}} f \stackrel{\text { def }}{=}\left\{\left\{f, F_{\mu}\right\}\right.$ for function $f$ (called Moyal adjoint), and $\delta$ denotes Kronecker's delta. It is noted here that the difference from the corresponding transformation of classical Hamiltonian systems is just the replacement of Poisson bracket by Moyal bracket in quantum systems.

Similarly to the previous studies, ${ }^{15-24,26,32-37,39,48-52}$ while two quantities $H_{0}^{(0)}$ and $H_{\mu}^{(\mu-1)}$ are known at the second equation $(v=\mu)$ of Eq. (59), the other two are not, implying that $F_{\mu}$ can be determined so that $H_{\mu}^{(\mu)}$ (and hence the new Hamiltonian $H^{(\mu)}$ ) has a "desired" form. Here we determine the generating function $F_{\mu}$ so that $H_{\mu}^{(\mu)}$ contain only those terms which commute with $I_{1}=x_{1} \xi_{1}$. Let the polynomial expression of $H_{\mu}^{(\mu-1)}$ be

$$
H_{\mu}^{(\mu-1)}=\sum_{j \boldsymbol{k}} h_{\mu, \boldsymbol{j} \boldsymbol{k}}^{(\mu-1)}(t) x_{1}{ }^{j_{1}} \cdots x_{n}{ }^{j_{n}} \xi_{1}{ }^{k_{1}} \cdots \xi_{n}{ }^{k_{n}},
$$

with the time-dependent coefficients $h_{\mu, j k}^{(\mu-1)}(t)$. Similarly, the generating function is expressed as

$$
F_{\mu}=\sum_{j k} f_{\mu, j k}^{(\mu-1)}(t) x_{1}^{j_{1}} \cdots x_{n}^{j_{n}} \xi_{1}^{k_{1}} \cdots \xi_{n}^{k_{n}} .
$$

Then the second equation $(\mu=\nu)$ of Eq. (59) is

$$
\begin{aligned}
H_{\mu}^{(\mu)}= & \sum_{j \boldsymbol{k}}\left(h_{\mu, \boldsymbol{j} \boldsymbol{k}}^{(\mu-1)}(t)+\gamma_{j \boldsymbol{k}} f_{\mu, \boldsymbol{j} \boldsymbol{k}}^{(\mu-1)}(t)-\frac{\mathrm{d}}{\mathrm{d} t} f_{\mu, \boldsymbol{j} \boldsymbol{k}}^{(\mu-1)}(t)\right) \\
& \times x_{1}{ }^{j_{1}} \cdots x_{n}{ }^{j_{n}} \xi_{1}{ }^{k_{1}} \cdots \xi_{n}{ }^{k_{n}},
\end{aligned}
$$

where

$$
\gamma_{j k} \stackrel{\text { def }}{=} \lambda\left(k_{1}-j_{1}\right)+\sum_{\ell=2}^{n} \mathrm{i} \omega_{\ell}\left(k_{\ell}-j_{\ell}\right)
$$

By setting [see Eq. (48)]

$$
f_{\mu, j k}^{(\mu-1)}(t)=S\left[\gamma_{j k}, h_{\mu, j k}^{(\mu-1)}\right](t)
$$

for $j_{1} \neq k_{1}$, terms with $j_{1} \neq k_{1}$ do not appear in $H_{\mu}^{(\mu)}$. Thus after the $N$ th-order transformation [Eq. (55)] we have

$H^{(N)}=\sum_{j \boldsymbol{k}} a_{\boldsymbol{j} \boldsymbol{k}}^{(N)}(t)\left(x_{1} \xi_{1}\right)^{j_{1}} x_{2}{ }^{j_{2}} \cdots x_{n}{ }^{j_{n}} \xi_{2}{ }^{k_{2}} \cdots \xi_{n}{ }^{k_{n}}+O\left(\varepsilon^{N}\right)$,

that is, all the terms below order $N$ in the transformed Hamiltonian $H^{(N)}$ contains the same power of $x_{1}$ and $\xi_{1}$, with the coefficients $a_{j k}^{(N)}(t)$. Then it can be proven that

$$
\left\{\left\{I_{1}, H^{(N)}\right\}=0,\right.
$$

where $I_{1}=x_{1} \xi_{1}$. Equation (66) can be verified by noting that the Moyal bracket is equal to the Poisson bracket when one of the arguments is a quadratic polynomial. ${ }^{57}$

The form of Eq. (65) is so-called partial NF, which has been found successful in a wide range of applications. ${ }^{15,17-20,23,25}$ We only cancel the terms with $j_{1} \neq k_{1}$, to make the Hamiltonian commute with $I_{1}$. In this case we always have nonzero real part in $\gamma_{j k}$, so that the definition of $S$ converges. Further normalization to make the Hamiltonian commute with the nonreactive mode actions $I_{\ell}(\ell=2, \ldots)$ is subject to so-called resonance problem. The resonance problem in the case of time-dependent systems discussed in Ref. 32 also holds for the quantum case considered in the present paper.

\section{Calculation of reactivity operator}

The reactivity operator is given by Eq. (39) with the transformation Eq. (55) obtained in Secs. II D 2 and II D 3. Since $\mathscr{P}_{0}=\Theta\left(x_{1}\right)$, the anharmonic reactivity operator is calculated through the Fourier transform of the Heaviside step function:

$$
\begin{aligned}
\mathscr{P} & =U^{*} \star \Theta\left(x_{1}\right) \star U, \\
& =U^{*} \star\left(\frac{1}{2}+\frac{1}{2 \pi} \mathrm{pp} \int \frac{1}{\mathrm{i} k} \exp \left(\mathrm{i} k x_{1}\right) \mathrm{d} k\right) \star U, \\
& =\frac{1}{2}+\frac{1}{2 \pi} \mathrm{pp} \int \frac{1}{\mathrm{i} k} U^{*} \star \exp \left(\mathrm{i} k x_{1}\right) \star U \mathrm{~d} k,
\end{aligned}
$$

where "pp" stands for the principal part. Now we use the fact that

$$
\exp \left(i k x_{1}\right)=\exp _{\star}\left(i k x_{1}\right)
$$

because higher derivatives in the star product all vanish for the linear function $x_{1}$, and

$$
U^{*} \star \exp _{\star}\left(\mathrm{i} k x_{1}\right) \star U=\exp _{\star}\left(\mathrm{i} k U^{*} \star x_{1} \star U\right),
$$

corresponding to the operator relation

$$
\hat{U}^{\dagger} \exp \left(\mathrm{i} k \hat{x}_{1}\right) \hat{U}=\exp \left(\mathrm{i} k \hat{U}^{\dagger} \hat{x}_{1} \hat{U}\right)
$$


Letting the transform of $x_{1}$ be

$$
\begin{aligned}
y_{1} & =y_{1}(\boldsymbol{x}, \boldsymbol{\xi}, t) \stackrel{\text { def }}{=} U^{*} \star x_{1} \star U \\
& =\exp \left(-\operatorname{Mad}_{F_{0}}\right) \exp \left(-\varepsilon \operatorname{Mad}_{F_{1}}\right) \cdots \exp \left(-\varepsilon^{N} \operatorname{Mad}_{F_{N}}\right) x_{1},
\end{aligned}
$$

Eq. (67) becomes

$$
\mathscr{P}=\frac{1}{2}+\frac{1}{2 \pi} \mathrm{pp} \int \frac{1}{\mathrm{i} k} \exp _{\star}\left(\mathrm{i} k y_{1}\right) \mathrm{d} k .
$$

The quantity $y_{1}$ defined in Eq. (71) is a function of the whole phase space coordinate $(\boldsymbol{x}, \boldsymbol{\xi})$ as well as time $t$. Hereafter we do not write the dependence explicitly. In the classical limit $\hbar \rightarrow 0, y_{1}$ converges to the previously found NF reaction coordinate whose sign uniquely tells the fate of the reaction in classical nonlinear systems. ${ }^{15,16,18-24,32,33}$ In the same limit the anharmonic reactivity operator [Eq. (72)] converges to a Heaviside step function of $y_{1}$, because the star-exponential becomes the usual exponential for $\hbar \rightarrow 0$. Without taking the classical limit, the star-exponential of $y_{1}$ is different from the usual exponential. For the calculation, we expand the correction term in $\mathrm{i} k$ :

$\exp _{\star}\left(\mathrm{i} k y_{1}\right)=\exp \left(\mathrm{i} k y_{1}\right)\left(1+\frac{(\mathrm{i} k)^{2}}{2 !} g_{2}+\frac{(\mathrm{i} k)^{3}}{3 !} g_{3}+\cdots\right)$,

where $g_{n}$ 's are also functions of $(\boldsymbol{x}, \boldsymbol{\xi}, t)$. It can be shown that the expansion starts with the quadratic term in $\mathrm{i} k$ (no linear term) and the coefficients are given by

$$
g_{n}=\sum_{m=0}^{n}\left(\begin{array}{c}
n \\
m
\end{array}\right)(\underbrace{y_{1} \star y_{1} \star \cdots \star y_{1}}_{m})\left(-y_{1}\right)^{n-m} .
$$

The expansion of Eq. (73) may need a comment since $\mathrm{i} k$ is not necessarily small. However, it is shown in Appendix that the function $g_{n}$ for large $n$ contains only high-order perturbations. More precisely, we have

$$
g_{n}=O\left(\varepsilon^{\lfloor(n+2) / 3\rfloor}\right),
$$

where $\lfloor r\rfloor$ is the largest integer that does not exceed $r$. Thus the truncation of the series in Eq. (73) at a certain order is validated.

Substituting Eq. (73) into Eq. (72), we obtain the expression for the reactivity operator

$$
\mathscr{P}=\Theta\left(y_{1}\right)+\frac{g_{2}}{2 !} \delta^{\prime}\left(y_{1}\right)+\frac{g_{3}}{3 !} \delta^{\prime \prime}\left(y_{1}\right)+\cdots .
$$

The first term corresponds to the classical picture that the sign of the nonlinear reaction coordinate $y_{1}$, which properly incorporates the effect of nonlinearities of the system and time-dependent external field, divides the whole space into two disjoint regions: all the phase space points with $y_{1}>0$ correspond to trajectories which will go to the product. However, the existence of the other terms in Eq. (76) tells that the reactivity in quantum anharmonic systems is determined also by derivatives of the distribution at the classical boundary $y_{1}=0$. [Note that $g_{n} \rightarrow 0$ as $\hbar \rightarrow 0$ since the starexponential in Eq. (73) becomes the usual exponential.] For an example which will be presented in Sec. III, however, it is found that the correction due to $g_{2}, g_{3}, \ldots$ is rather minor compared to the difference between $\Theta\left(y_{1}\right)$ and $\Theta\left(x_{1}\right)$ (harmonic approximation).

Note that the convergence of the series expansions used in the present theory is not always guaranteed. In the series of works on the NF in the time-independent systems ${ }^{15,17-20,23,25}$ it has been shown that this method is useful up to moderately high energy: the form of Eq. (65) is so-called partial NF, which avoids the notorious problem of resonance among the nonreactive modes and the laser field, ${ }^{32}$ occurring as the energy increases above the saddle point energy, and therefore applicable to a wide region up to moderately high energy. The convergence range can be further extended by reducing the degree of normalization. ${ }^{39}$ Investigating quantum analog of it would be an interesting future subject. In numerical applications, the convergence must be checked for each system. The way of checking the convergence will be presented in Sec. III B by using a numerical example.

\section{NUMERICAL ILLUSTRATION}

\section{A. Model system}

To illustrate how the theory in Sec. II interprets quantum dynamics of reactions under the influence of optimally designed laser fields, we analyze a simple one-dimensional system under a laser field, which was regarded as mimicking an isomerization of a molecule. ${ }^{65}$

The Hamiltonian is given by

$$
H=\frac{p^{2}}{2 m}+V(q)+\mathcal{E}(t) \mu(q)
$$

with the effective mass $m=99.33 \mathrm{amu}$, the potential energy

$$
V(q)=-\frac{m \lambda^{2}}{2} q^{2}+a_{3} q^{3}+a_{4} q^{4}
$$

$m \lambda^{2} / 2=3.79 \mathrm{~cm}^{-1} \AA^{-2}, a_{3}=1.59 \mathrm{~cm}^{-1} \AA^{-3}, a_{4}=9.22$ $\mathrm{cm}^{-1} \AA^{-4}$, the time-dependent electric field $\mathcal{E}(t)$, and the dipole moment

$$
\mu(q)=\mu_{1} q
$$

with $\mu_{1}=0.301 e$. The potential energy, energy eigenvalues, and eigenfunctions without the electric field are shown in

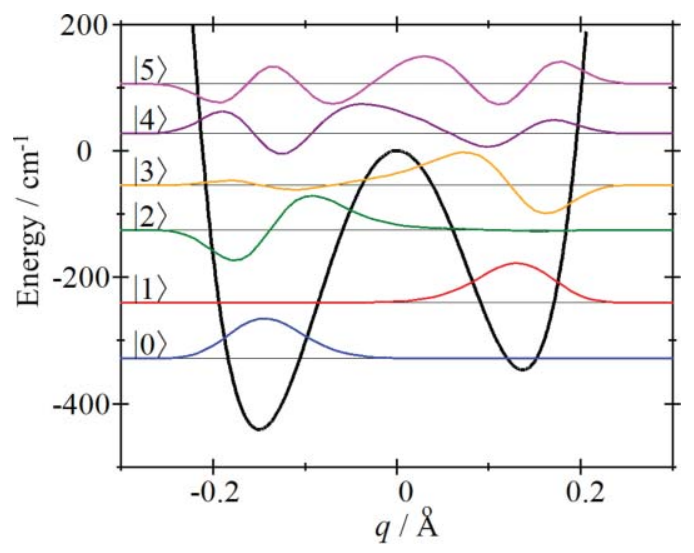

FIG. 2. Potential energy, energy eigenvalues, and eigenfunctions of the model system. 
Fig. 2. Throughout the numerical calculations, we use the sinc-function discrete variable representation of Colbert and Miller, ${ }^{66}$ where the interval of $q \in[-0.4 \AA, 0.4 \AA]$ is divided into 80 equally spaced grid points.

The laser field was designed, by using the local control theory in Ref. 65 , to populate the first excited state $|1\rangle$ as much as possible, starting with $100 \%$ initial population in the ground state $|0\rangle$.

Figure 3 shows (a) the resultant electric field obtained by the optimal control, and (b) the change of the population in each state when the wavefunction is propagated by the Schrödinger equation with the electric field shown in (a). The final population of $95 \%$ in the state $|1\rangle$ was achieved. The whole process can be interpreted as the excitation in the left well $(|0\rangle \rightarrow|2\rangle)$, the passage over the barrier $(|2\rangle \rightarrow|3\rangle$, or $|2\rangle \rightarrow|4\rangle \rightarrow|3\rangle$ ), and the de-excitation in the right well $(|3\rangle \rightarrow|1\rangle)$. The populations in the states $|5\rangle$ and higher remain minor.

The question to be addressed here is why the laser field to guide the reaction was optimized as in Fig. 3(a) and, in other words, whether we can provide a sound physical interpretation based on the underlying phase space structure. To achieve this, we investigate the phase space structure by ap-

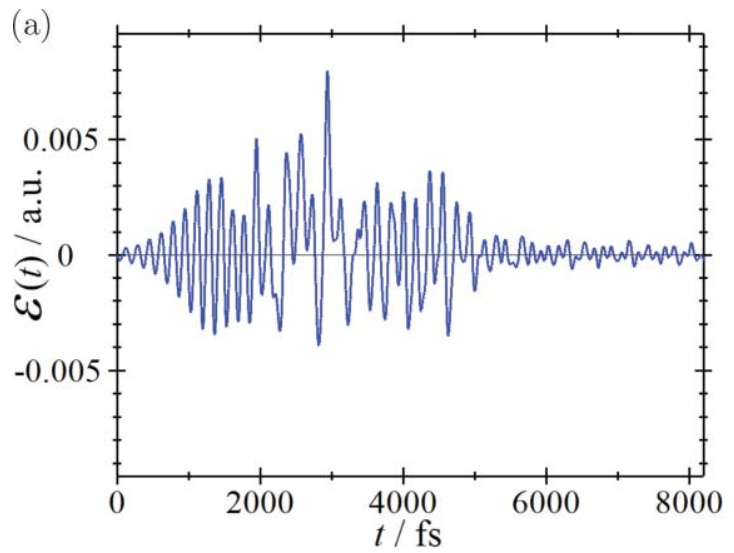

(b)

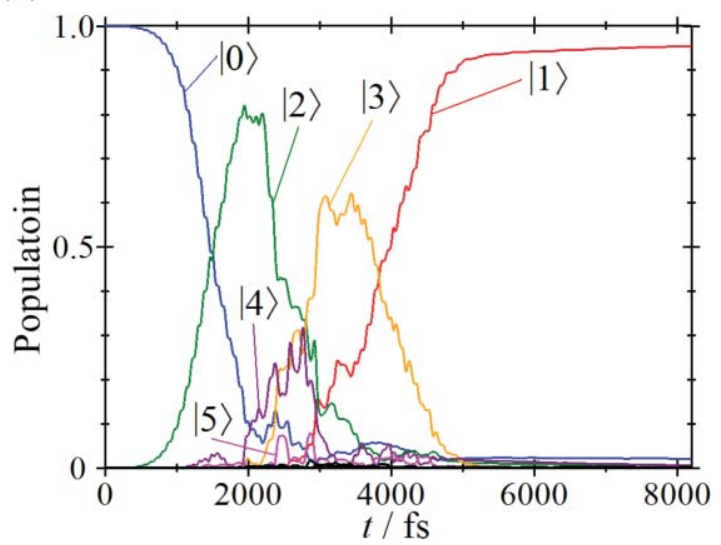

FIG. 3. Results of optimal control. (a) Electric field as a function of time. To obtain the optimal field, we used the local control theory presented in Ref. 65, with the laser intensity parameter $I=6 \mathrm{TW} / \mathrm{cm}^{2}$, the weight $(\boldsymbol{q}$ or $\boldsymbol{F}$ in their notation) being $(1,0.75,0.5,0.25,0)$ for state $0,2,4,3,1$, respectively, and the laser penalty factor $R=1 \mathrm{ps}^{-1}$. (b) Change of the population in each state. plying the normal form theory presented in Sec. II. Following Eqs. (40)-(43), we decompose the Hamiltonian as

$$
\begin{aligned}
H & =H_{0}+H_{1}+H_{2}, \\
H_{0} & =\frac{1}{2 m} p^{2}-\frac{m \lambda^{2}}{2} q^{2}+\mu_{1} \mathcal{E}(t) q, \\
H_{1} & =a_{3} q^{3}, \\
H_{2} & =a_{4} q^{4} .
\end{aligned}
$$

The skewed coordinates are introduced by [Eq. (2)]

$$
x=\frac{m \lambda q+p}{\sqrt{2 m \lambda}}, \quad \xi=\frac{p-m \lambda q}{\sqrt{2 m \lambda}},
$$

so that the lowest order becomes

$$
H_{0}=\lambda x \xi+\frac{\mu_{1}}{\sqrt{2 m \lambda}} \mathcal{E}(t)(x-\xi)
$$

corresponding to Eq. (43). We can now follow the procedure of Sec. II to obtain the normal form transformation and the projection operator. The perturbation calculation up to the second order is performed in the present example. This order will be shown to be accurate enough in Sec. III B.

\section{B. Accuracy check of quantum normal form}

As seen in Sec. II, the reaction probability $P_{\text {reaction }}$ is represented in terms of anharmonic reactivity operator $\hat{\mathscr{P}}(t)$ :

$$
P_{\text {reaction }}=\langle\psi(t)|\hat{\mathscr{P}}(t)| \psi(t)\rangle .
$$

Due to the fact that it is a projection onto the eigenstates, the time derivative should vanish for $\hat{\mathscr{P}}(t)$ :

$$
\frac{\mathrm{d}}{\mathrm{d} t}\langle\psi(t)|\hat{\mathscr{P}}(t)| \psi(t)\rangle=\left\langle\psi(t)\left|\frac{\mathrm{d} \hat{\mathscr{P}}(t)}{\mathrm{d} t}\right| \psi(t)\right\rangle=0,
$$

where the time derivative of an operator is defined as

$$
\frac{\mathrm{d} \hat{\mathscr{P}}(t)}{\mathrm{d} t} \stackrel{\text { def }}{=} \frac{1}{\mathrm{i} \hbar}[\hat{\mathscr{P}}(t), \hat{H}(t)]+\frac{\partial \hat{\mathscr{P}}(t)}{\partial t} .
$$

In practice, however, we cannot calculate Eq. (84) because the NF theory is based on the perturbation series in the vicinity of the saddle point. Therefore to confine our analyses in the saddle region, we introduce a localization operator $\hat{\sigma}$ defined by

$$
\hat{\sigma}=\Theta\left(q-q_{1}\right)-\Theta\left(q-q_{2}\right),
$$

(i.e, $\hat{\sigma}=1$ for $q_{1}<q<q_{2}$ and 0 otherwise). We evaluate the following quantity

$$
\left\langle\hat{\sigma} \frac{\mathrm{d} \hat{\mathscr{P}}(t)}{\mathrm{d} t} \hat{\sigma}\right\rangle \stackrel{\text { def }}{=}\left\langle\psi(t)\left|\hat{\sigma} \frac{\mathrm{d} \hat{\mathscr{P}}(t)}{\mathrm{d} t} \hat{\sigma}\right| \psi(t)\right\rangle .
$$

In this model system we choose $q_{1}=-0.1 \AA$ and $q_{2}=0.1 \AA$. Equation (87) should be close to 0 if $\hat{\mathscr{P}}(t)$ is actually a good approximation to the true reactivity operator in the region $q_{1}<q<q_{2}$. Therefore, the value of Eq. (87) serves as a check on the accuracy of our NF calculation.

The next question is how to judge whether the value of Eq. (87) is "small." We must compare the value with a certain 
reference which should have the typical scale of the quantity in consideration. We now note that Eq. (87) has the physical dimension of flux, and can indeed be interpreted (at least approximately) as a flux in the phase space going through the boundary (separatrix) $y_{1}=0$. The "typical value" of the flux for this system can be estimated by the flux through the surfaces $q=q_{j}(j=1,2),\left\langle\hat{f}_{j}\right\rangle$ given by

$$
\begin{aligned}
\left\langle\hat{f}_{j}\right\rangle & \stackrel{\text { def }}{=}\left\langle\psi(t)\left|\hat{f}_{j}\right| \psi(t)\right\rangle \\
& =\left\langle\psi(t)\left|\frac{\hat{p} \delta\left(q-q_{j}\right)+\delta\left(q-q_{j}\right) \hat{p}}{2 m}\right| \psi(t)\right\rangle .
\end{aligned}
$$

Figure 4(a) shows the fluxes through the surfaces $q=q_{1}$ and $q=q_{2}$, and the quantity of Eq. (87) with the wavefunction evolving under the optimal laser field in Fig. 3(a). Note that the values $q_{1}=-0.1 \AA$ and $q_{2}=0.1 \AA$ are relatively large in size, in fact closer to the minima than the saddle point. [The minima are located at $q=-0.150 \AA$ and $q=0.137 \AA$ (see Fig. 2).] The oscillatory behavior of the flux $\left\langle\hat{f}_{1}\right\rangle$ with relatively large amplitude across $q_{1}=-0.1 \AA$ before $\sim 2900$ fs represents the system residing and exciting vibrationally in the reactant well [cf. Fig. 2 and the major

(a)

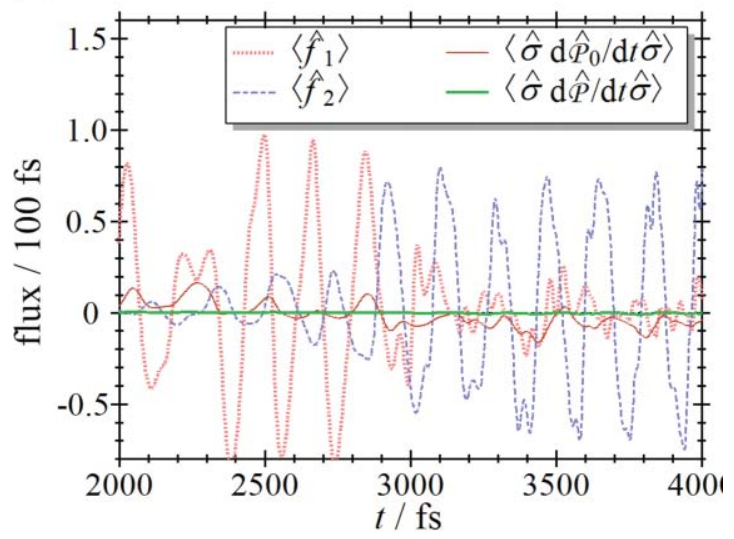

(b)

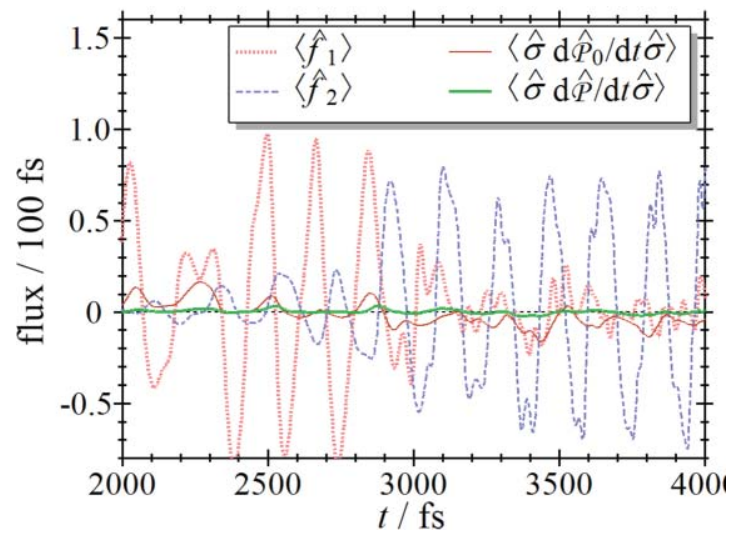

FIG. 4. (a) Fluxes through the surfaces $q=q_{1}, q=q_{2}$, and the separatrices obtained by the harmonic approximation without the laser field and by the second-order NF calculation. Dotted: $\left\langle\hat{f}_{1}\right\rangle$. Dashed: $\left\langle\hat{f}_{2}\right\rangle$. Thin solid: $\left\langle\hat{\sigma}\left(\mathrm{d} \hat{\mathscr{P}}_{0}(t) / \mathrm{d} t\right) \hat{\sigma}\right\rangle$. Bold solid: $\langle\hat{\sigma}(\mathrm{d} \hat{\mathscr{P}} / \mathrm{d} t) \hat{\sigma}\rangle$. The last is almost identical with the horizontal axis (flux $=0$ ). (b) Same with (a), but $\hat{\mathscr{P}}$ is calculated by the step function $\mathrm{Op}\left[\Theta\left(y_{1}\right)\right]$. population dynamics of $|0\rangle \rightarrow|2\rangle$ in Fig. 3(b)]. Likewise, that of $\left\langle\hat{f}_{2}\right\rangle$ across $q_{1}=0.1 \AA$ after $\sim 2900$ fs represents the system being captured in the product well like $|3\rangle \rightarrow|1\rangle$. In the figure, $\langle\hat{\sigma}(\mathrm{d} \hat{\mathscr{P}}(t) / \mathrm{d} t) \hat{\sigma}\rangle$ is plotted in which the NF perturbation calculation was performed up to the second order, and the coefficients up to $g_{4}$ was evaluated in the expansion of Eq. (76), together with $\left\langle\hat{\sigma}\left(\mathrm{d} \hat{\mathscr{P}}_{0}(t) / \mathrm{d} t\right) \hat{\sigma}\right\rangle$ for comparison. Compared with the typical values of the fluxes $\left\langle\hat{f}_{1}\right\rangle$ and $\left\langle\hat{f}_{2}\right\rangle$, the time derivative of $\hat{\mathscr{P}}_{0}=\mathrm{Op}\left[\Theta\left(x_{1}\right)\right]$ in the harmonic approximation does not vanish in the region $q_{1}<q<q_{2}$ (extracted by $\hat{\sigma}$ ). This implies that the surface of $x_{1}=0$ does not serve as a proper reaction boundary.

In turn, when we use the reactivity operator $\hat{\mathscr{P}}$ calculated by the second-order NF, its time derivative becomes very small and the plot is almost identical with the horizontal axis within the resolution of flux in the figure. The result implies that the operator $\hat{\mathscr{P}}$ can actually extract the reactive part of the system in the region of $q_{1}<q<q_{2}$. Although the NF calculation is based on the expansion around the saddle point, the convergence range is large considering that $q_{1}=-0.1 \AA$ is actually closer to the minima than the saddle point. As soon as the system crosses the surface $q_{1}=-0.1 \AA$ inside the reactant well, we can precisely identify whether it will go over to the product side or come back to the reactant side, by the use of the NF reactivity operator.

To estimate the effect of the deviation from the simple step-function behavior of the reactivity operator Eq. (76), we plot in Fig. 4(b) the results of the same calculation with the NF reactivity operator approximated by the step function $\hat{\mathscr{P}} \approx \mathrm{Op}\left[\Theta\left(y_{1}\right)\right]$ [i.e., only the first term in Eq. (76)]. In this case, the result of NF shows some residual error, but it remains minor compared to the change from $\Theta\left(x_{1}\right)$ to $\Theta\left(y_{1}\right)$.

\section{Movement of separatrix and reaction control}

Next we explore how the anharmonic reactivity operator can provide physical interpretation of the process of reaction control by laser. As discussed in Fig. 4, the transition from the reactant to the product seems to occur around $2900 \mathrm{fs}$. In order to capture the underlying physical mechanism of the reaction, we plot the Wigner distributions at two time instants 2824 and 2912 fs in Fig. 5. One can see in Fig. 4 that, around 2824 fs, the system is going into the saddle region through $q=q_{1}$ (i.e., the positive $\left\langle\hat{f}_{1}\right\rangle$ ), and around $2912 \mathrm{fs},\left\langle\hat{f}_{1}\right\rangle$ becomes negative and smaller in magnitude, and $\left\langle\hat{f}_{2}\right\rangle$ becomes, in turn, positive and larger in magnitude. This implies that, around $2912 \mathrm{fs}$, some components of the system (passing through the surface $q=q_{1}$ ) go out through $q=q_{2}$ and some goes back through $q=q_{1}$. Then, the component once reflected back into the reactant around 2912 fs oscillates in the well and "tries again" to enter the saddle region through $q=q_{1}$, as seen from the oscillatory behavior with smaller amplitude in $\left\langle\hat{f}_{1}\right\rangle$ after $2912 \mathrm{fs}$. The two time instant $t=2824$ and 2912 fs can be regarded as the representative time instants before and after the major reaction event.

Figure 5(a) shows the Wigner distribution at $t=2824 \mathrm{fs}$ in the phase space $(p, q)$ together with the separatrix $y_{1}=0$ obtained by the second-order NF (solid orange curves), and 
(a) $t=2824 \mathrm{fs}$

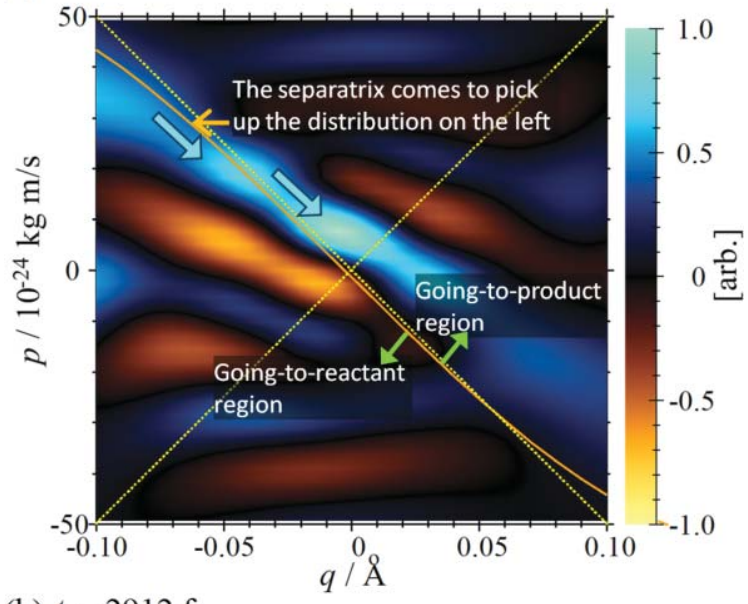

(b) $t=2912 \mathrm{fs}$

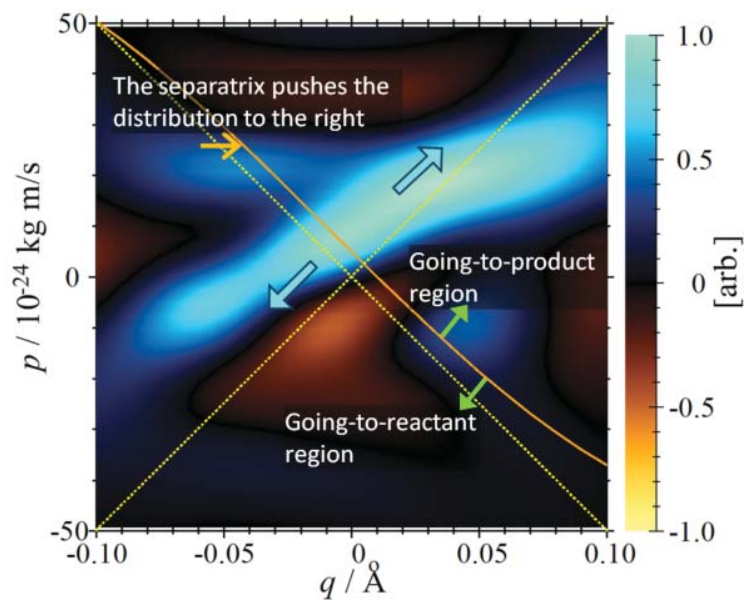

FIG. 5. Snapshots of the Wigner distributions at (a) $t=2824 \mathrm{fs}$, where the system is entering into the saddle region from the upper left, and (b) $t$ $=2912 \mathrm{fs}$, where the system is going toward the product region in the upper right. Dotted curves show the zeroth-order separatrix $x_{1}=0$. Solid curves show the separatrix $y_{1}=0$ calculated by the second-order NF. Blue bold arrows roughly indicate the direction in which the distribution is moving. Small orange arrows show the migration of the separatrix compared to the zeroth-order counterpart, with the physical implications written in insets. The destinations of the phase space flow (going-to-product or going-to-reactant) divided by the separatrix are indicated by the green arrows.

the harmonic approximation $x_{1}=0$ (dotted yellow lines). Compared to the harmonic approximation, the separatrix $y_{1}=0$ has migrated a little to the left by the effect of the (optimally controlled) laser field. Since the upper part of the phase space divided by the separatrix $y_{1}=0$ corresponds to all the reactive (going-to-the-product) trajectories, this migration of the separatrix is interpreted as increasing the reaction probability by "catching" the system excited in the reactant.

The Wigner distribution and the separatrix $y_{1}=0$ at $t=2912 \mathrm{fs}$ are shown in Fig. 5(b). The separatrix $y_{1}=0$ modulated by the laser field is found to have migrated to the opposite (right) side in the phase space $(p, q)$. As discussed with Fig. 4, the surface $y_{1}=0$ serves as the boundary which the system cannot cross, this migration of the separatrix is interpreted as pushing the system toward the product. In summary, it is found that the separatrix, moving with time due to the time-dependence of the Hamiltonian (the laser field), "catches" the system coming from the left and "releases" it toward the product region.

\section{SUMMARY AND OUTLOOK}

Recent developments of the theory of saddle point dynamics are extended to quantum mechanical systems propagating under time-dependent external fields. As the theory is based on the Weyl expressions and the Wigner distribution, interpretation is facilitated by its classical phase space picture, while the exact quantum mechanical values can be calculated.

In terms of a new unitary transformation which incorporates the effect of nonlinearity and time-dependence, the Hamiltonian is transformed into a so-called normal form that commutes with the action operator. This enables us to introduce a quantum reactivity operator, which precisely extracts the reactive parts from the phase space of the system. Its Weyl representation as a phase space function identifies the reactive region in the phase space. Either in quantum harmonic systems or in classical (anharmonic) systems, the Weyl representation of the reactivity is a Heaviside step function, being unity in the reactive region and zero in the nonreactive region. In quantum anharmonic systems, however, it turned out to involve a high-order derivatives of the delta function at the surface $y_{1}=0$ in addition to the step function $\Theta\left(y_{1}\right)$. In timedependent systems, the boundary $y_{1}=0$ between the reactive and nonreactive parts of the phase space moves with time. It was found in a simple one-dimensional double-well system that the migration of the reaction boundary arising from the optimally controlled laser pulse "catches" the system exciting in the reactant and "releases" it into the product.

Although the model system studied in this paper was a one-dimensional system, the theory is formulated generally for multidimensional systems. An application of the present theory to laser-controlled multidimensional reacting system would be interesting. In multidimensional systems, motions along the vibrational modes may affect the reaction due to the nonlinear couplings. However, as in classical anharmonic systems $^{15,16,18-24,32,33}$ the present theory is expected to disentangle these couplings and identify the true reaction coordinate $\left(y_{1}\right)$ to tell us the fate of the reaction in quantum systems. Analytical expression of this reaction coordinate obtained by the present theory can elucidate how the couplings and the fields affect the reactivity, by similar analyses in Refs. 34 and 35.

It is also interesting to note that the optimal field is not necessarily unique. For example, pulse shapes obtained by the optimal control methods $\mathrm{s}^{1,3,8-10}$ depends on the penalty factor to the laser intensity. This can in general be made time-dependent, ${ }^{11}$ constraining the pulse envelop. Frequency constraint ${ }^{12-14}$ can be also incorporated to restrict the spectral components of the pulse. Different inputs in these conditions lead to different pulse shapes, with possibly different mechanisms of the reaction. It would be interesting to systematically look into these mechanisms in terms of the present theory. 


\section{ACKNOWLEDGMENTS}

This work has been supported by Research Fellowships of the Japan Society for the Promotion of Science for Young Scientists (to SK) and by JSPS, JST/CREST, Priority Area "Molecular Theory for Real Systems" (to TK). The computations were partially performed using the Research Center for Computational Science, Okazaki, Japan.

\section{APPENDIX: PROOF OF EQ. (75)}

In Eq. (73), expansion in terms of $i k$ was performed as

$$
\exp _{\star}\left(\mathrm{i} k y_{1}\right) \exp \left(-\mathrm{i} k y_{1}\right)=\sum_{n=0}^{\infty} \frac{(\mathrm{i} k)^{n}}{n !} g_{n} .
$$

We first note that $y_{1}$ is expanded in perturbation series:

$$
y_{1}=y_{1}^{(0)}+\varepsilon y_{1}^{(1)}+\varepsilon^{2} y_{1}^{(2)}+\cdots,
$$

with

$$
y_{1}^{(0)}=x_{1} .
$$

Let

$$
y_{1}=x_{1}+w,
$$

that is,

$$
w=\varepsilon y_{1}^{(1)}+\varepsilon^{2} y_{1}^{(2)}+\cdots .
$$

We start by considering the following quantity:

$$
Z \stackrel{\text { def }}{=} \exp \left(-\frac{\mathrm{i} k x_{1}}{2}\right) \star \exp _{\star}\left(\mathrm{i} k y_{1}\right) \star \exp \left(-\frac{\mathrm{i} k x_{1}}{2}\right) \text {. }
$$

Noting that the liner canonical transformation does not change the form of the operator $\Lambda$ in the exact formula for the star product [Eq. (11)],

$$
\Lambda=\overleftarrow{\partial_{q}} \cdot \overrightarrow{\partial_{p}}-\overleftarrow{\partial_{p}} \cdot \overrightarrow{\partial_{q}}=\overleftarrow{\partial_{x}} \cdot \overrightarrow{\partial_{\xi}}-\overleftarrow{\partial_{\xi}} \cdot \overrightarrow{\partial_{x}}
$$

we have

$$
\begin{aligned}
& \exp \left(\mathrm{i} k x_{1}\right) \star f(\boldsymbol{x}, \boldsymbol{\xi}) \\
& \quad=\exp \left(\mathrm{i} k x_{1}\right) \exp (\mathrm{i} \hbar \Lambda / 2) f(\boldsymbol{x}, \boldsymbol{\xi}) \\
& =\exp \left(\mathrm{i} k x_{1}\right) \exp \left(\frac{-k \hbar}{2} \frac{\partial}{\partial \xi_{1}}\right) f(\boldsymbol{x}, \boldsymbol{\xi}) \\
& =\exp \left(\mathrm{i} k x_{1}\right) f\left(\boldsymbol{x}, \xi_{1}-k \hbar / 2, \xi_{2}, \ldots\right),
\end{aligned}
$$

and similarly

$$
\begin{aligned}
& f(\boldsymbol{x}, \boldsymbol{\xi}) \star \exp \left(\mathrm{i} k x_{1}\right) \\
& \quad=\exp \left(\mathrm{i} k x_{1}\right) f\left(\boldsymbol{x}, \xi_{1}+k \hbar / 2, \xi_{2}, \ldots\right),
\end{aligned}
$$

for any smooth function $f(\boldsymbol{x}, \boldsymbol{\xi})$. Therefore,

$$
Z=\exp _{\star}\left(\mathrm{i} k y_{1}\right) \exp \left(-\mathrm{i} k x_{1}\right),
$$

and the left hand side of Eq. (A1) is given by $Z \exp (-\mathrm{i} k w)$.

We now expand $Z$ in terms of $\mathrm{i} k$,

$$
Z=\sum_{n=0}^{\infty}(\mathrm{i} k)^{n} C_{n}
$$

and prove $C_{n}=O\left(\varepsilon^{\lfloor(n+2) / 3\rfloor}\right)$ by induction. In the expansion of $\exp (-\mathrm{i} k w)$, the power of $\varepsilon$ in each term is always greater than the power of $k$ because of Eq. (A5). Combining these two facts it is shown that the power of $\varepsilon$ is greater than the power of $k$ in the expansion of $Z \exp (-\mathrm{i} k w)$, completing the proof of Eq. (75).

By differentiating Eq. (A6) we have

$$
\begin{aligned}
\frac{\mathrm{d} Z}{\mathrm{~d}(\mathrm{i} k)}= & \exp \left(-\frac{\mathrm{i} k x_{1}}{2}\right) \star \frac{w}{2} \star \exp \left(\frac{\mathrm{i} k x_{1}}{2}\right) \star Z \\
& +Z \star \exp \left(\frac{\mathrm{i} k x_{1}}{2}\right) \star \frac{w}{2} \star \exp \left(-\frac{\mathrm{i} k x_{1}}{2}\right),
\end{aligned}
$$

where we have used

$$
\begin{aligned}
\frac{\mathrm{d}}{\mathrm{d}(\mathrm{i} k)} \exp _{\star}\left(\mathrm{i} k y_{1}\right) & =y_{1} \star \exp _{\star}\left(\mathrm{i} k y_{1}\right)=\exp _{\star}\left(\mathrm{i} k y_{1}\right) \star y_{1} \\
& =\frac{1}{2}\left(y_{1} \star \exp _{\star}\left(\mathrm{i} k y_{1}\right)+\exp _{\star}\left(\mathrm{i} k y_{1}\right) \star y_{1}\right)
\end{aligned}
$$

because $y_{1}$ commutes with $\exp _{\star}\left(\mathrm{i} k y_{1}\right)$. By using the Moyal adjoint, we can write

$$
\begin{aligned}
\frac{\mathrm{d} Z}{\mathrm{~d}(\mathrm{i} k)}= & \left(\exp \left(-\frac{k \hbar}{2} \operatorname{Mad}_{x_{1}}\right) \frac{w}{2}\right) \star Z \\
& +Z \star\left(\exp \left(\frac{k \hbar}{2} \operatorname{Mad}_{x_{1}}\right) \frac{w}{2}\right) .
\end{aligned}
$$

Equating each order of $k$ we have

$$
\begin{aligned}
(n+1) C_{n+1}= & \sum_{m=0}^{n} \frac{1}{m !}\left[\left(\left(\frac{\mathrm{i} \hbar}{2} \operatorname{Mad}_{x_{1}}\right)^{m} \frac{w}{2}\right) \star C_{n-m}\right. \\
& \left.+C_{n-m} \star\left(\left(-\frac{\mathrm{i} \hbar}{2} \operatorname{Mad}_{x_{1}}\right)^{m} \frac{w}{2}\right)\right],
\end{aligned}
$$

which is a recursion formula to give $C_{n+1}$ in terms of $C_{m}$ 's with $0 \leq m \leq n$. Since $w=\sum_{v=1}^{\infty} \varepsilon^{v} y_{1}^{(v)}$, we have

$$
\left(\frac{\mathrm{i} \hbar}{2} \operatorname{Mad}_{x_{1}}\right)^{m} \frac{w}{2}=\sum_{\nu=1}^{\infty} \varepsilon^{\nu}\left(\frac{\mathrm{i} \hbar}{2} \operatorname{Mad}_{x_{1}}\right)^{m} \frac{y_{1}^{(\nu)}}{2} .
$$

Now we recall that $y_{1}^{(v)}$ is a polynomial of degree $1+v$, and that the operation of $\operatorname{Mad}_{x_{1}}$ reduces the polynomial degree by 1 . The polynomial degree of the $v$ th term in Eq. (A16) is therefore $1+v-m$. When the terms do not vanish, they must satisfy $1+v-m \geq 0$. This means that only the terms with $v \geq m-1$ appear in the summation of Eq. (A16), and therefore Eq. (A16) is $O\left(\varepsilon^{\max (1, m-1)}\right)$, where $\max (1, m-1)$ is the larger value of 1 and $m-1$. Putting this and the hypothesis of the induction $\left[C_{m}=O\left(\varepsilon^{\lfloor(m+2) / 3\rfloor}\right)\right.$ for $\left.0 \leq \forall m \leq n\right]$ into Eq. (A15), we obtain $C_{n+1}=O\left(\varepsilon^{\lfloor(n+3) / 3\rfloor}\right)$.

${ }^{1}$ S. A. Rice and M. Zhao, Optical Control of Molecular Dynamics (Wiley, New York, 2000).

${ }^{2}$ Molecules in Laser Fields, edited by A. D. Bandrauk (Marcel Dekker, New York, 1994).

${ }^{3}$ T. Brabec and F. Krausz, Rev. Mod. Phys. 72, 545 (2000).

${ }^{4}$ R. J. Levis, G. M. Menkir, and H. Rabitz, Science 292, 709 (2001).

${ }^{5}$ K. Yamanouchi, Science 295, 1659 (2002).

${ }^{6}$ C. Daniel, J. Full, L. González, C. Lupulescu, J. Manz, S. V. A. Merli, and L. Wöste, Science 299, 536 (2003). 
${ }^{7}$ B. J. Sussman, D. Townsend, M. Y. Ivanov, and A. Stolow, Science 314, 278 (2006).

${ }^{8}$ R. Kosloff, S. A. Rice, P. Gaspard, S. Tersigni, and D. J. Tannor, Chem. Phys. 139, 201 (1989).

${ }^{9}$ W. Zhu, J. Botina, and H. Rabitz, J. Chem. Phys. 108, 1953 (1998).

${ }^{10}$ W. Zhu and H. Rabitz, J. Chem. Phys. 109, 385 (1998).

${ }^{11}$ K. Sundermann and R. de Vivie-Riedle, J. Chem. Phys. 110, 1896 (1999).

${ }^{12}$ J. Werschnik and E. K. U. Gross, J. Opt. B 7, S300 (2005).

${ }^{13}$ J. Werschnik and E. K. U. Gross, J. Phys. B 40, R175 (2007).

${ }^{14}$ M. Lapert, R. Tehini, G. Turinici, and D. Sugny, Phys. Rev. A 79, 063411 (2009).

${ }^{15}$ Geometrical Structures of Phase Space in Multidimensional Chaos: Applications to Chemical Reaction Dynamics in Complex Systems, edited by M. Toda, T. Komatsuzaki, T. Konishi, R. S. Berry, and S. A. Rice, Advances in Chemical Physics, Vols. 130A and 130B (John-Wiley \& Sons, New York, 2005), and references therein.

${ }^{16}$ Advancing Theory for Kinetics and Dynamics of Complex, ManyDimensional Systems: Clusters and Proteins, edited by T. Komatsuzaki, R. S. Berry, and D. M. Leitner, Advances in Chemical Physics, Vol. 145 (John-Wiley \& Sons, New York, in press).

${ }^{17}$ R. Hernandez and W. H. Miller, Chem. Phys. Lett. 214, 129 (1993).

${ }^{18}$ R. Hernandez, J. Chem. Phys. 101, 9534 (1994).

${ }^{19}$ T. Komatsuzaki and R. S. Berry, J. Chem. Phys. 110, 9160 (1999).

${ }^{20}$ T. Komatsuzaki and R. S. Berry, Proc. Natl. Acad. Sci. U.S.A. 98, 7666 (2001).

${ }^{21}$ S. Wiggins, L. Wiesenfeld, C. Jaffé, and T. Uzer, Phys. Rev. Lett. 86, 5478 (2001).

${ }^{22}$ T. Uzer, C. Jaffé, J. Palacián, P. Yanguas, and S. Wiggins, Nonlinearity 15, 957 (2002).

${ }^{23}$ T. Komatsuzaki and R. S. Berry, Adv. Chem. Phys. 123, 79 (2002).

${ }^{24}$ S. Kawai, H. Teramoto, C.-B. Li, T. Komatsuzaki, and M. Toda, "Dynamical reaction theory based on geometric structures in phase space," Adv. Chem. Phys. (in press).

${ }^{25}$ C.-B. Li, A. Shojiguchi, M. Toda, and T. Komatsuzaki, Few-Body Syst. 38, 173 (2006).

${ }^{26}$ C.-B. Li, A. Shojiguchi, M. Toda, and T. Komatsuzaki, Phys. Rev. Lett. 97, 028302 (2006).

${ }^{27}$ T. Bartsch, R. Hernandez, and T. Uzer, Phys. Rev. Lett. 95, 058301 (2005).

${ }^{28}$ T. Bartsch, T. Uzer, and R. Hernandez, J. Chem. Phys. 123, 204102 (2005).

${ }^{29}$ T. Bartsch, T. Uzer, J. M. Moix, and R. Hernandez, J. Chem. Phys. 124, 244310 (2006).

${ }^{30}$ T. Bartsch, J. Chem. Phys. 131, 124121 (2009).

${ }^{31}$ R. Hernandez, T. Uzer, and T. Bartsch, Chem. Phys. 370, 270 (2010).

${ }^{32}$ S. Kawai, A. D. Bandrauk, C. Jaffé, T. Bartsch, J. Palacián, and T. Uzer, J. Chem. Phys. 126, 164306 (2007).
${ }^{33}$ T. Bartsch, J. M. Moix, R. Hernandez, S. Kawai, and T. Uzer, Adv. Chem. Phys. 140, 191 (2008).

${ }^{34}$ S. Kawai and T. Komatsuzaki, J. Chem. Phys. 131, 224505 (2009).

${ }^{35}$ S. Kawai and T. Komatsuzaki, J. Chem. Phys. 131, 224506 (2009).

${ }^{36}$ S. Kawai and T. Komatsuzaki, Phys. Chem. Chem. Phys. 12, 7626 (2010).

${ }^{37}$ S. Kawai and T. Komatsuzaki, Phys. Chem. Chem. Phys. 12, 7636 (2010).

${ }^{38}$ S. Kawai and T. Komatsuzaki, Phys. Chem. Chem. Phys. 12, 15382 (2010).

${ }^{39}$ S. Kawai and T. Komatsuzaki, Phys. Rev. Lett. 105, 048304 (2010).

${ }^{40}$ H. Eyring, J. Chem. Phys. 3, 107 (1935).

${ }^{41}$ E. Wigner, J. Chem. Phys. 5, 720 (1937).

${ }^{42}$ M. G. Evans and M. Polanyi, Trans. Faraday Soc. 31, 875 (1935).

${ }^{43}$ O. K. Rice and H. C. Ramsperger, J. Am. Chem. Soc. 50, 617 (1928).

${ }^{44}$ L. S. Kassel, J. Phys. Chem. 32, 1065 (1928).

${ }^{45}$ R. A. Marcus, J. Chem. Phys. 20, 359 (1952).

${ }^{46}$ J. C. Keck, Adv. Chem. Phys. 13, 85 (1967).

${ }^{47}$ D. G. Truhlar and B. C. Garrett, Acc. Chem. Res. 13, 440 (1980).

${ }^{48}$ T. Komatsuzaki and M. Nagaoka, J. Chem. Phys. 105, 10838 (1996).

${ }^{49}$ T. Komatsuzaki and M. Nagaoka, Chem. Phys. Lett. 265, 91 (1997).

${ }^{50} \mathrm{H}$. Waalkens, A. Burbanks, and S. Wiggins, J. Chem. Phys. 121, 6207 (2004).

${ }^{51}$ C.-B. Li, Y. Matsunaga, M. Toda, and T. Komatsuzaki, J. Chem. Phys. 123, 184301 (2005).

${ }^{52}$ C. Jaffe, S. D. Ross, M. W. Lo, J. Marsden, D. Farrelly, and T. Uzer, Phys. Rev. Lett. 89, 011101 (2002).

${ }^{53}$ L. E. Fried and G. S. Ezra, J. Chem. Phys. 86, 6270 (1987).

${ }^{54}$ L. E. Fried and G. S. Ezra, J. Comp. Chem. 8, 397 (1987).

${ }^{55}$ G. S. Ezra, C. C. Martens, and L. E. Fried, J. Phys. Chem. 91, 3721 (1987).

${ }^{56}$ L. E. Fried and G. S. Ezra, J. Phys. Chem. 92, 3144 (1988).

${ }^{57}$ H. Waalkens, R. Schubert, and S. Wiggins, Nonlinearity 21, R1 (2008).

${ }^{58}$ A. Goussev, R. Schubert, H. Waalkens, and S. Wiggins, J. Chem. Phys. 131, 144103 (2009).

${ }^{59}$ T. Seideman and W. H. Miller, J. Chem. Phys. 95, 1768 (1991).

${ }^{60}$ C. K. Zachos, D. B. Fairlie, and T. L. Curtright, Quantum Mechanics in Phase Space (World Scientific, Singapore, 2005).

${ }^{61}$ R. G. Parr and W. Yang, Density-Functional Theory of Atoms and Molecules (Oxford University Press, New York, 1989).

${ }^{62}$ N. L. Balazs and A. Voros, Ann. Phys. 199, 123 (1990).

${ }^{63}$ M. Abramowitz and I. A. Stegun, Handbook of Mathematical Functions (Dover, New York, 1965).

${ }^{64}$ W. H. Miller, J. Chem. Phys. 61, 1823 (1974).

${ }^{65}$ M. Sugawara and Y. Fujimura, J. Chem. Phys. 100, 5646 (1994).

${ }^{66}$ D. T. Colbert and W. H. Miller, J. Chem. Phys. 96, 1982 (1992). 